\title{
9. PETROGRAPHY AND PROVENANCE OF VOLCANICLASTIC SANDS RECOVERED FROM THE IZU-BONIN ARC, LEG $126^{1}$
}

\author{
Kathleen M. Marsaglia ${ }^{2}$
}

\begin{abstract}
Modal compositions of volcaniclastic sands recovered on Leg 126 of the Ocean Drilling Project (Izu-Bonin island arc and Sumisu Rift) are similar to those from other intraoceanic island arcs and associated marginal basins. These sands are dominantly composed of volcanic-lithic and plagioclase-feldspar grains derived from the Izu-Bonin magmatic arc and intrarift volcanoes. The glass color of volcanic fragments ranges from black (tachylite) to brown to colorless; individual samples usually contain a mixture of glass colors. Two of the forearc sites (792 and 793) are more heterogeneous with respect to glass color than the backarc/Sumisu Rift sites $(788,790$, and 791$)$. Site 787 forearc sands are dominantly composed of tachylite grains; their unique composition may be attributed either to winnowing by submarine-canyon currents or to a volcanic island source. There is an increase in the proportions of pumice/colorless glass, felsitic grains, and quartz within sediments of the incipient backarc basin (Sumisu Rift), as compared with the forearc-basin sites.
\end{abstract}

\section{INTRODUCTION}

The Izu-Bonin intraoceanic arc, located on Iwo Jima Ridge, is a northward extension of the Mariana arc system (Fig. 1). A series of holes were drilled during Ocean Drilling Program (ODP) Leg 126 in the Izu-Bonin forearc basin and across the arc into the Sumisu Rift (Figs. 2 and 3), an incipient backarc basin produced by rifting west of the Izu-Bonin Arc (Brown and Taylor, 1988). On previous Deep Sea Drilling Project (DSDP) legs, the coarse-grained volcaniclastic aprons flanking volcanic arcs created penetration problems, resulting in only a few successful DSDP sites located along or adjacent to active arc axes (see Nishimura et al., 1991, for further discussion). On Leg 126, however, thick volcaniclastic sections were successfully penetrated by piston coring in the Sumisu Rift, on the rift flank, and within the forearc basin (Taylor, Fujioka, et al., 1990). Core containing unconsolidated and consolidated volcaniclastic sand was recovered at six sites (Figs. 1, 2, and 3): two sites in the rift, one near the center of the eastern half graben of the rift (Site 791), and the other above the flank of a nearby basement high (Site 790); one site atop the eastern flank of the rift basin (Site 788); and three sites in the forearc basin of the Izu-Bonin Arc (Sites 787, 792, and 793). Sediments recovered on Leg 126 provide excellent recent analogs for possible ancient intraoceanic-arc deposits.

Over 200 volcanic centers exist in and around the Sumisu Rift (Brown and Taylor, 1988), ranging in composition from tholeiitic basalt to sodic rhyolite (Fryer et al., 1985). The Sumisu Rift basin is in a position to receive both frontal-arc-derived sediment and sediment derived from the chain of volcanoes that separate the northern and southern parts of the Sumisu Rift basin (Brown and Taylor, 1988). Nishimura and Murakami (1988) found that volcaniclastic components in the upper meter of basin fill were most likely supplied by Quaternary mafic to dacitic eruptions of the Sumisu Jima (submarine, north end of rift) and Torishima (subaerial, south end of rift) frontalarc volcanoes (Fig. 2), with little contribution from intrarift submarine volcanoes. Brown and Taylor (1988) and Taylor, Fujioka, et al. (1990) also propose that the volcaniclastic sand in the Sumisu Rift was likely derived from episodic eruptions of arc volcanoes. Brown and Taylor

\footnotetext{
${ }^{1}$ Taylor, B., Fujioka, K., et al., 1992. Proc. ODP, Sci. Results, 126: College Station, TX (Ocean Drilling Program).

${ }^{2}$ Department of Geological Sciences, The University of Texas at El Paso, El Paso, TX 79968, U.S.A
}

(1988), Nishimura and Murakami (1988), and Taylor, Fujioka, et al. (1990) interpret the layered clastic units recovered in cores on the Sumisu basin floor to be the products of turbidity currents and gravity-flow processes. Another possible source of volcaniclastic detritus is reworked material eroded from the uplifted eastern flank

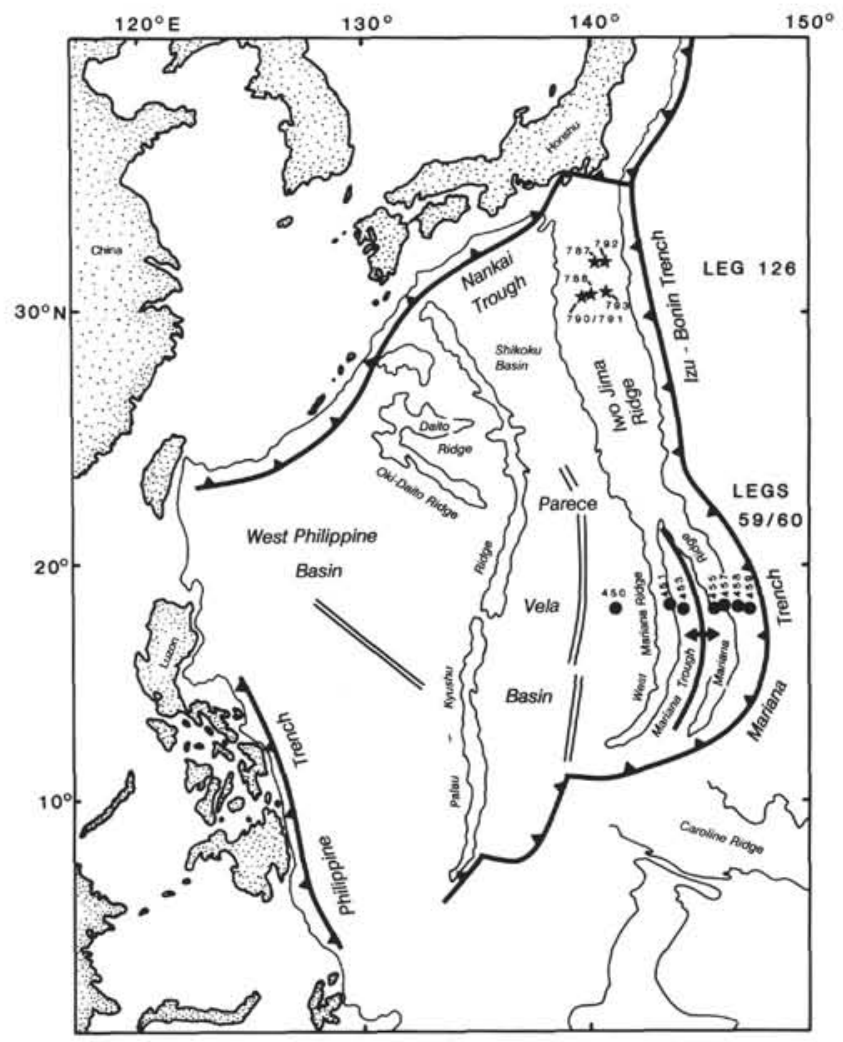

Figure 1. Tectonic map of the west-central Pacific Ocean, showing locations of Mariana transect sites (DSDP Legs 59 and 60) and Leg 126 sites. Subduction zones are indicated by barbed heavy lines, active spreading ridges by heavy lines with arrows, and inactive spreading ridges by parallel lines. Modified from Natland and Tarney (1981). 


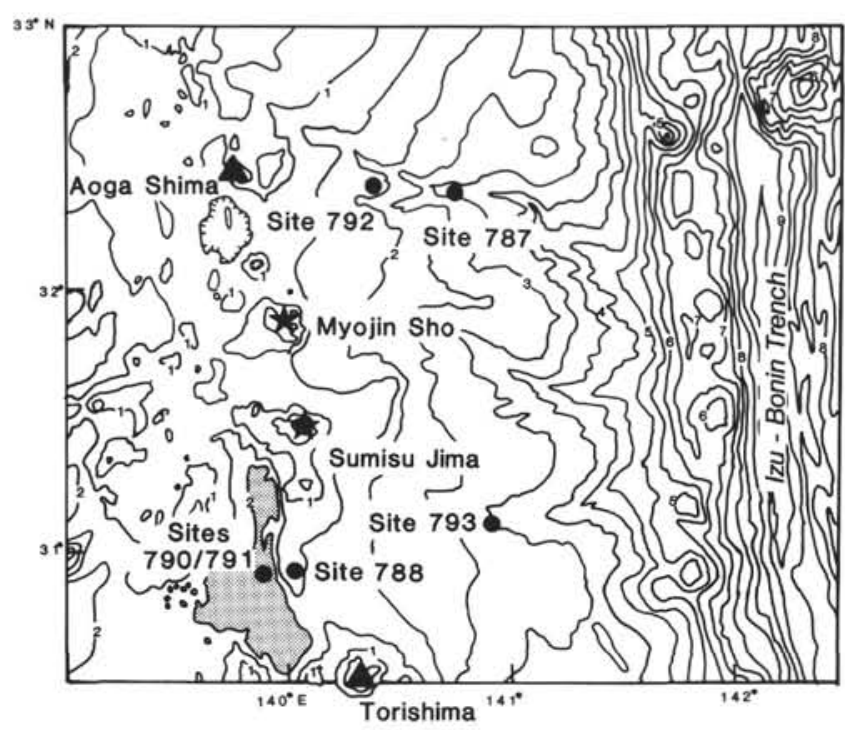

Figure 2. Bathymetric map of the Izu-Bonin arc-trench system illustrating Leg 126 site locations, major arc volcanoes (stars $=$ submergent volcanoes and triangles $=$ volcanic islands), and the Sumisu Rift (shaded region in lower left). The contour interval is $0.5 \mathrm{~km}$. Modified from Taylor, Fujioka, et al. (1990).

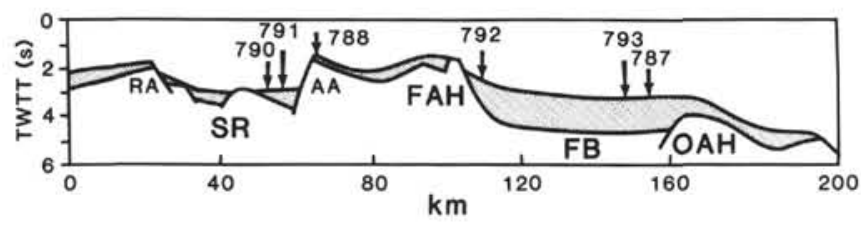

Figure 3. Simplified, schematic cross-section across the Izu-Bonin Arc, with Leg 126 site locations indicated by arrows. Modified from Leg 126 Shipboard Scientific Party (1989). TWTT = two-way traveltime, $S R=$ Sumisu Rift, FAH = forearc high, $\mathrm{FB}=$ forearc basin, $\mathrm{OAH}=$ outer-arc high, $\mathrm{AA}=\operatorname{arc}$ axis, and $\mathrm{RA}=$ remnant arc.

of the rift, where Sites 788 and 789 are located (Taylor, Fujioka, et al., 1990).

The purposes of this investigation are (1) to characterize the modal sand composition of unconsolidated/minimally altered volcaniclastic sands recovered on Leg 126; (2) to compare the composition of sands recovered in the Izu-Bonin forearc with those recovered in the Sumisu intra-arc rift (across the Izu-Bonin Arc); and (3) to compare the composition of volcaniclastic sands from the Izu-Bonin arc transect (an incipient backarc basin) with the data of Packer and Ingersoll (1986) for volcaniclastic sands recovered on a transect across the Mariana Arc and backarc basin (Fig. 1).

\section{METHODS}

Cores containing unconsolidated sandy sediment recovered at Sites 787, 788, 790, 791, 792, and 793 were sampled for this study. These unconsolidated samples were first air-dried and then lightly sieved by hand for the sand fraction $(0.0625-2.0 \mathrm{~mm})$. Sand concentrates were then epoxied to glass slides and ground to $30 \mu \mathrm{m}$ in thickness. All thin sections were subsequently stained for both calcium- and potassium-rich feldspars (see appendix in Marsaglia and Tazaki, this volume). Because trends in tertiary parameters (e.g., lithic category subdivisions) were examined in this study, higher precision for smaller quantities required more total points (Van der Plas and
Tobi, 1965). Where possible, 500 (or more where bioclasts are present) points were counted on each section using the Gazzi-Dickinson method (Dickinson, 1970; Ingersoll et al., 1984), thus minimizing dependence of composition on grain size. Grid spacings were used that maximized coverage of the thin section and were larger than the maximum grain size. The Gazzi-Dickinson method also divides volcanic lithic clasts into various textural types (Dickinson, 1970), which is especially significant in magmatic-arc settings, where volcaniclastic components may constitute up to $100 \%$ of the sand fraction. In addition, grains were separated according to glass color under planepolarized light (i.e., orange, $\tan$ /brown, black, and colorless).

Counted grains were placed into 47 monomineralic and polymineralic compositional categories. Point-count categories and recalculated parameters are defined in Table 1 and discussed below. Raw point-count data are presented in Table 2 , recalculated parameters in Table 3, and site means and standard deviations in Table 4. Recalculated parameters, means, and standard deviations were calculated using a Lotus 123 spreadsheet program.

\section{GRAIN TYPES}

In the volcaniclastic sands recovered on Leg 126, monocrystalline grains are dominantly plagioclase feldspar and nonopaque ferromagnesian minerals, both as separate grains and as sand-size crystals in volcanic lithic fragments (Plate 1, Figs. 1 and 2). Only a few samples contain trace amounts of potassium feldspar, and roughly half the samples contain traces of monocrystalline quartz. Rare, enigmatic, unstained, untwinned framework silicate grains with first-order gray to white birefringence and exhibiting biaxial optic figures may be sodium-rich feldspar (albite) or slightly strained quartz. Because of this uncertainty, these grains were not placed in either feldspar or quartz totals in recalculating parameters.

Opaque dense minerals were counted but not differentiated. Nonopaque dense minerals include pyroxene (and/or olivine), epidote, and rare amphibole (Sample 126-788C-4H-4, 46-49 cm), which was included in the pyrobole/olivine total. The high concentrations of fine diagenetic opaque minerals observed in some thin sections may be related to hydrothermal alteration (e.g., Samples 126-788C-7H-1, $30-33 \mathrm{~cm}$, and $126-790 \mathrm{C}-16 \mathrm{X}-5,66-69 \mathrm{~cm})$, and rare grains of pyroxene exhibiting cockscomb texture imply minor grain dissolution (e.g., Samples 126-791A-5H-2, 92-95 cm, and 126-790B-10H$6,119-120 \mathrm{~cm})$.

Lithic fragments in Leg 126 samples are, with few exceptions, volcanic; in pyroclastic terminology, the morphologies and textures of these volcanic grains vary from blocky to bubble wall to pumice (Fisher and Schmincke, 1984). Using the Gazzi-Dickinson method, the volcanic lithic fragments are subdivided into vitric, felsitic (Plate 1, Fig. 3), microlitic, lathwork (Dickinson, 1970), and "other" categories (Table 1). In addition, the first three were divided according to glass color and texture or alteration as viewed in plane-polarized light (Table 1).

Glassy fragments were divided into colorless, brown (including tan, brown, and greenish brown), and black (tachylite) varieties. These subdivisions are related to provenance because glass color is thought to be a function of composition and cooling rate (Schmincke, 1981). Schmincke (1981) determined refractive indices of Leg 60 glass shards and documented a direct relationship among glass color, refractive index, and composition: colorless glasses have lower refractive indices and higher silica contents; light brown or green glasses have intermediate refractive indices and silica contents; and dark-brown glasses have high refractive indices and low silica contents. Fragments of basaltic glass include sideromelane (transparent tan to brown in transmitted light) and tachylite (opaque in transmitted light because of the presence of $\mathrm{Fe} / \mathrm{Ti}$-rich microlites) (Fisher and Schmincke, 1984). The presence of sideromelane is ascribed to high 
Table 1. Counted and recalculated parameters.

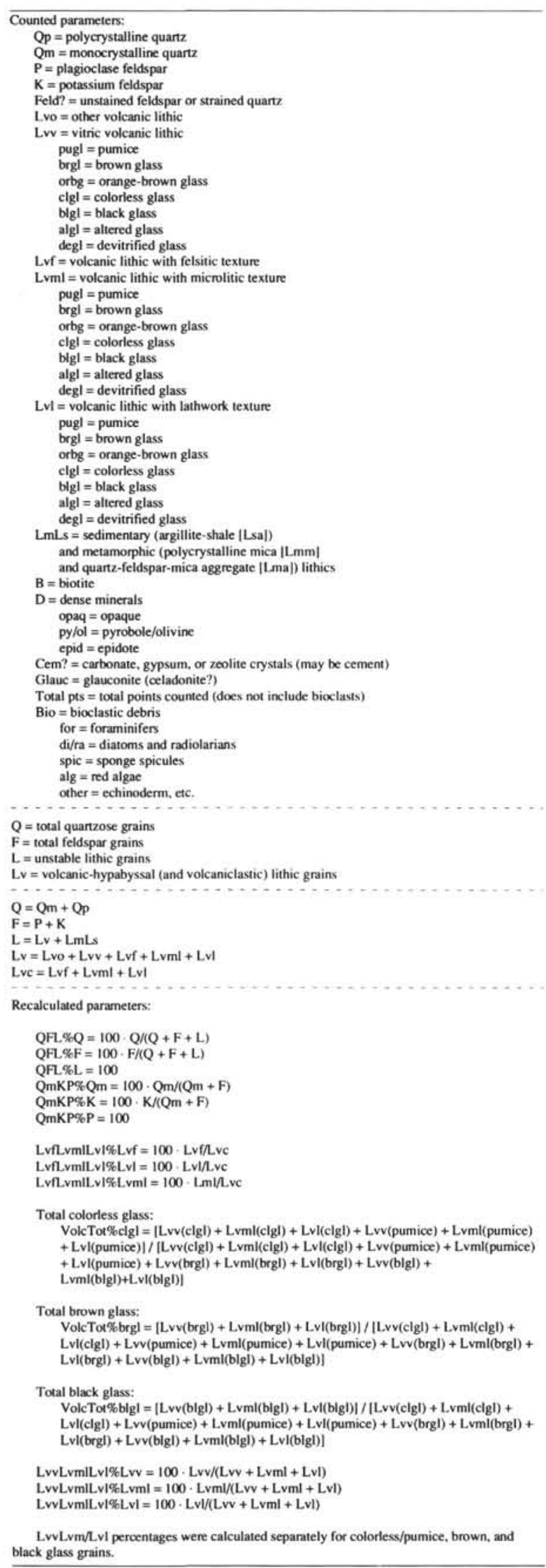

cooling rates induced by quenching of magma by water, whereas tachylitic glass is formed in subaerial or subaqueous conditions where cooling rates are lower (Macdonald, 1972; Fisher and Schmincke, 1984; Cas and Wright, 1987).

The vitric category (Lvv; Table 1) includes fragments of colorless pumice (Plate 1,Fig. 4), colorless glass shards and bubble-wall fragments (Plate 2, Fig. 1), tan/brown glass with variable vesicularity (Plate 2, Fig. 2 ), and black or dark gray glass (tachylite) with variable vesicularity (Plate 2, Fig. 3). These vitric fragments are characterized by their lack of microlites. Minor vitric grain categories include orange/brown glass (variable morphology), glass altered to clay minerals (Plate 2, Fig. 4), and devitrified glass. Greenish brown glass (e.g., Samples 126-790B-7H-6, 86-90 cm; 126-792A-1H-6, 95-99 cm; 126-792E1R-1, 92-96 cm; 126-792E-9R-3, 68-73 cm; and 126-792E-28R-2, $94-97 \mathrm{~cm}$ ) is included in the brown category. In some of the samples (e.g., 126-790C-12X-1, 81-84 cm; 126-790C-15X-CC, 12-14 cm; 126-790C-20X-5, 31-34 cm; 126-791A-5H-2, 92-95 cm; 126-792B$8 \mathrm{X}-3,62-65 \mathrm{~cm}$; and 126-792E-9R-5, 26-31 cm), incipient devitrification is indicated by low birefringence of glass fragments, but these were still counted as vitric components. Locally, within a thin section, the colors of glass were gradational either from colorless to tan to brown (e.g., Samples 126-788A-4R-1, 18-21 cm; 126-788C-3H-1, $42-46 \mathrm{~cm} ; 126-788 \mathrm{C}-11 \mathrm{H}-3,125-128 \mathrm{~cm} ; 126-788 \mathrm{C}-14 \mathrm{H}-5,57-60$ cm; 126-790A-2H-5, 9-12 cm; 126-790C-11X-4, 44-47 cm; 126$792 \mathrm{~A}-2 \mathrm{H}-4,46-50 \mathrm{~cm}$; and 126-792B-5X-CC, $25-28 \mathrm{~cm}$ ) or from brown to black (e.g., Samples 126-790A-2H-6, 106-107 cm, and 126-792B-5X-CC, 25-28 cm) (Plate 3, Fig. 1). In some instances, the color appeared related to the density of vesicles in fragments (e.g., Sample 126-790A-2H-5, 9-12 cm) with pumiceous fragments colorless and less vesicular fragments tan to brown.

Microlitic volcanic lithic fragments (Lvml; Table 1) contain variable amounts of microlites of feldspar and ferromagnesian minerals visible at high magnification. These microlites are primarily silt-size plagioclase or pyroxene crystals. Lathwork volcanic lithic fragments (Lvl; Table 1) are glassy fragments that contain sand-size microlites. A grain is counted as lathwork only if the cross hairs land on the glassy groundmass (e.g., Ingersoll et al., 1984). Microlitic and lathwork grains were subdivided into categories based on the color and texture of their glassy groundmass in plane-polarized light (colorless pumice: Plate 3, Fig. 2; colorless glass: Plate 1, Fig. 1; tan/brown glass: Plate 1, Fig. 2; Plate 2, Fig. 2, and Plate 3, Figs. 1 and 3; black glass: Plate 3, Fig. 4, and Plate 4, Fig. 1; orange glass, glass altered to clay minerals, and devitrified glass). Phenocrysts in lathwork fragments include plagioclase feldspar, pyroxene, and opaque minerals.

The "other volcanic lithic" category (Lvo; Table 1) includes combinations of crystals not covered by the Dickinson (1970) categories. These other volcanic grains form only a small percentage of arc-related volcaniclastic sand, in general (Marsaglia, 1989), and constitute only a few percent of the grains in the volcaniclastic sediments recovered on Leg 126 . This category includes holocrystalline aggregates of (1) plagioclase and opaque, (2) opaque and pyroxene, (3) pyroxene and plagioclase, and (4) pyroxene, plagioclase, and opaque (Plate 4, Fig. 2).

Other minor constituent grains include (1) "dirty" chert fragments (Qp), which may be altered or devitrified vitric grains, or perhaps silicified (hydrothermally altered?) argillaceous or glass-rich matrix; (2) glauconitic grains (may be celadonitized glass); (3) argillite-shale sedimentary lithic fragments (which may be argillaceous matrix not totally disaggregated by sieving) and metamorphic lithic fragments (both in LmLs category); and (4) authigenic crystals of zeolite, carbonate or gypsum cement (not counted in grain total).

Bioclasts were also counted but not included in the 500-point total (Table 2). Bioclast categories include foraminifer, diatom/radiolarian, sponge spicule, red algae, and other (e.g., fragments of pelecypods, echinoderms, bryozoans, etc.). Many samples contain no bioclastic debris. 
Table 2. Raw point-count data.

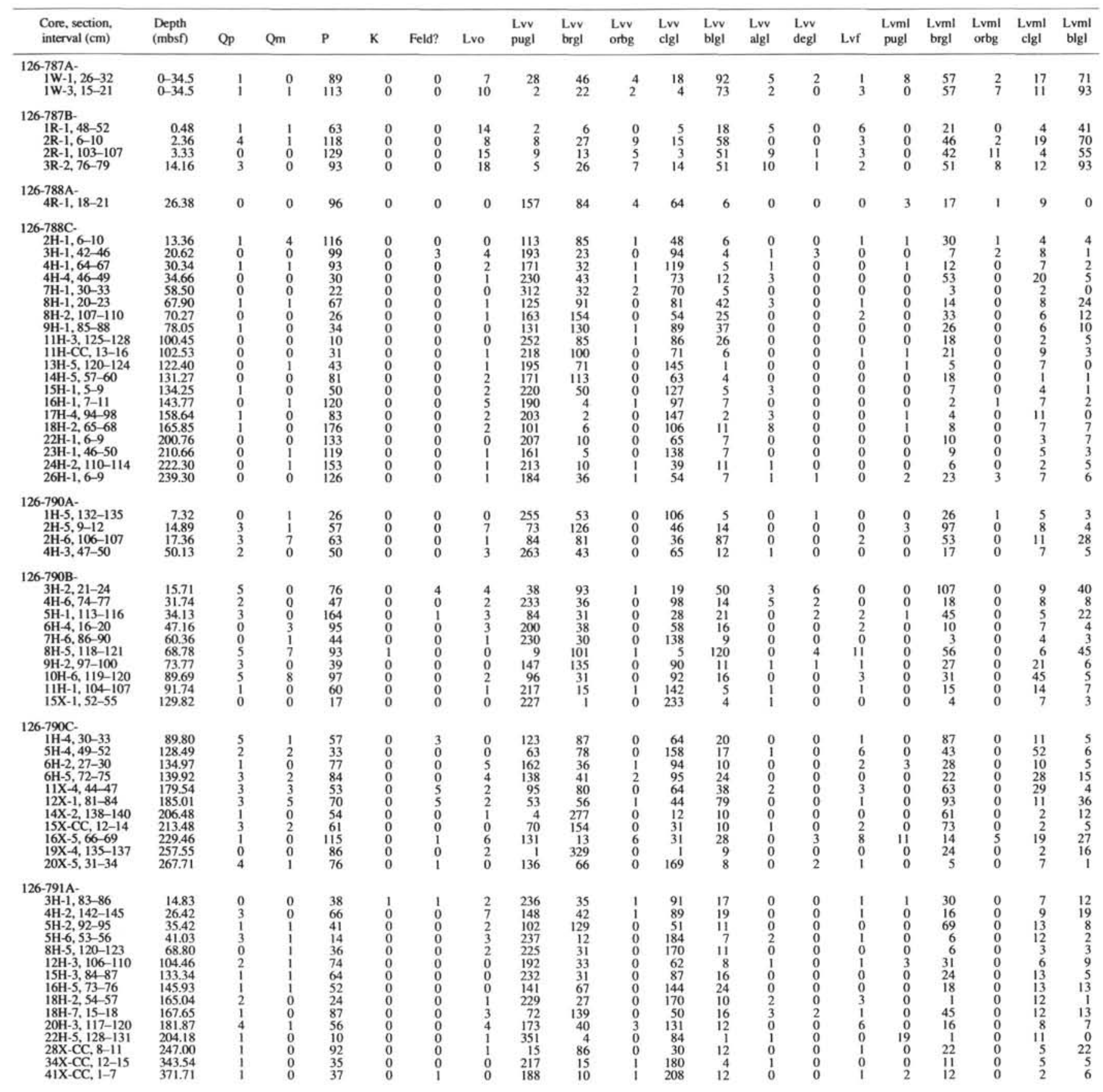

\section{SAND DETRITAL MODES}

On a QFL triangular diagram, all of the Leg 126 volcaniclastic sands examined in this study plot in the "Undissected Arc Field" of Dickinson et al. (1983). These sands are composed of predominantly feldspar and volcanic lithic grains, with negligible percentages of quartz (Fig. 4). Other plots of recalculated parameters, such as LvLsLm and QmKP diagrams, are not shown because of the uniformity of the data. Leg 126 sands would plot on or near the volcanic apex on an LvLsLm diagram, with virtually no variation among sites. Most samples also plot near the plagioclase apex on a QmKP diagram; however, some samples at Sites 790, 791, 792, and 793 are slightly enriched in quartz (Table 3). The general modal compositions of Leg 126 volcaniclastic sands are similar to those associated with other intraoceanic volcanic arcs (Marsaglia, 1989).

Differences exist among the sites with respect to the types of microcrystalline volcanic lithic fragments (e.g., felsitic [Lvf], microlitic [Lvml], and lathwork [Lvl]) present (see Marsaglia, 1991, for a further discussion of the significance of microcrystalline volcanic lithic proportions). An LvfLvmlLvl ternary plot of backarc and forearc site samples (Fig. 5) shows that the forearc samples cluster toward the Lvml apex, whereas some sands from Sites 790 and 791 are slightly enriched 
Table 2 (continued).

\begin{tabular}{|c|c|c|c|c|c|c|c|c|c|c|c|c|c|c|c|c|c|c|c|c|c|}
\hline $\begin{array}{l}\text { Lvml } \\
\text { algl }\end{array}$ & $\begin{array}{l}\text { Lvml } \\
\text { degl }\end{array}$ & $\begin{array}{l}\text { Lv1 } \\
\text { pugl }\end{array}$ & $\begin{array}{l}\text { Lvl } \\
\text { brgl }\end{array}$ & $\begin{array}{l}\text { Lvl } \\
\text { orbg }\end{array}$ & $\begin{array}{l}\text { Lvl } \\
\text { clgl }\end{array}$ & $\begin{array}{l}\text { Lvl } \\
\text { blg1 }\end{array}$ & $\begin{array}{l}\text { LvI } \\
\text { algl }\end{array}$ & $\begin{array}{l}\text { Lvl } \\
\text { degl }\end{array}$ & LmLs & B & $\underset{\text { opaq }}{\text { D }}$ & $\underset{\mathrm{ol} / \mathrm{py}}{\mathrm{D}}$ & $\begin{array}{c}\text { D } \\
\text { epid }\end{array}$ & Cement? & Glauc & $\begin{array}{c}\text { Total } \\
\text { pts }\end{array}$ & $\begin{array}{l}\text { Bio } \\
\text { for }\end{array}$ & $\begin{array}{c}\text { Bio } \\
\text { di/ra }\end{array}$ & $\begin{array}{l}\text { Bio } \\
\text { spic }\end{array}$ & $\begin{array}{l}\text { Bio } \\
\text { alg }\end{array}$ & $\begin{array}{l}\text { Bio } \\
\text { other }\end{array}$ \\
\hline$\frac{1}{2}$ & $\begin{array}{l}0 \\
0\end{array}$ & $\begin{array}{l}0 \\
0\end{array}$ & $\begin{array}{r}17 \\
8\end{array}$ & i & $\begin{array}{l}2 \\
5\end{array}$ & $\begin{array}{l}12 \\
36\end{array}$ & $\begin{array}{l}2 \\
0\end{array}$ & $\begin{array}{l}0 \\
0\end{array}$ & $\begin{array}{l}0 \\
0\end{array}$ & $\begin{array}{l}0 \\
0\end{array}$ & $\begin{array}{l}1 \\
3\end{array}$ & $\begin{array}{l}16 \\
44\end{array}$ & $\begin{array}{l}0 \\
0\end{array}$ & $\begin{array}{l}0 \\
0\end{array}$ & $\begin{array}{l}0 \\
0\end{array}$ & $\begin{array}{l}500 \\
500\end{array}$ & $\begin{array}{r}40 \\
3\end{array}$ & $\begin{array}{l}0 \\
0\end{array}$ & $\begin{array}{l}3 \\
0\end{array}$ & $\begin{array}{l}0 \\
1\end{array}$ & $\begin{array}{r}14 \\
5\end{array}$ \\
\hline $\begin{array}{l}0 \\
5 \\
3 \\
3\end{array}$ & $\begin{array}{l}0 \\
1 \\
0 \\
0\end{array}$ & $\begin{array}{l}0 \\
1 \\
1 \\
0\end{array}$ & $\begin{array}{r}7 \\
19 \\
15 \\
16\end{array}$ & $\begin{array}{l}0 \\
1 \\
6 \\
2\end{array}$ & $\begin{array}{l}3 \\
7 \\
2 \\
2\end{array}$ & $\begin{array}{l}34 \\
42 \\
26 \\
34\end{array}$ & $\begin{array}{l}1 \\
1 \\
2 \\
5\end{array}$ & $\begin{array}{l}0 \\
0 \\
1 \\
0\end{array}$ & $\begin{array}{l}0 \\
0 \\
0 \\
0\end{array}$ & $\begin{array}{l}0 \\
0 \\
0 \\
0\end{array}$ & $\begin{array}{r}2 \\
3 \\
14 \\
13\end{array}$ & $\begin{array}{l}16 \\
32 \\
80 \\
31\end{array}$ & $\begin{array}{l}0 \\
0 \\
0 \\
0\end{array}$ & $\begin{array}{l}0 \\
0 \\
0 \\
0\end{array}$ & $\begin{array}{l}0 \\
0 \\
0 \\
0\end{array}$ & $\begin{array}{l}250 \\
500 \\
500 \\
500\end{array}$ & $\begin{array}{r}0 \\
18 \\
1 \\
13\end{array}$ & $\begin{array}{l}0 \\
0 \\
0 \\
0\end{array}$ & $\begin{array}{l}0 \\
0 \\
0 \\
0\end{array}$ & $\begin{array}{l}0 \\
2 \\
1 \\
1\end{array}$ & $\begin{array}{r}2 \\
9 \\
6 \\
10\end{array}$ \\
\hline 0 & 0 & 1 & 8 & 0 & 5 & 0 & 0 & 0 & 0 & 0 & 24 & 21 & 0 & 0 & 0 & 500 & 0 & 0 & 0 & 0 & 0 \\
\hline $\begin{array}{l}0 \\
0 \\
0 \\
1 \\
0 \\
0 \\
0 \\
0 \\
0 \\
0 \\
0 \\
0 \\
2 \\
0 \\
0 \\
0 \\
0 \\
0 \\
0 \\
0\end{array}$ & $\begin{array}{l}0 \\
0 \\
0 \\
0 \\
0 \\
0 \\
0 \\
0 \\
0 \\
0 \\
0 \\
0 \\
0 \\
0 \\
0 \\
0 \\
0 \\
0 \\
0 \\
0\end{array}$ & $\begin{array}{l}3 \\
1 \\
1 \\
0 \\
0 \\
0 \\
0 \\
0 \\
0 \\
1 \\
0 \\
0 \\
0 \\
0 \\
5 \\
1 \\
4 \\
1 \\
1\end{array}$ & $\begin{array}{r}6 \\
5 \\
3 \\
7 \\
7 \\
0 \\
7 \\
5 \\
6 \\
6 \\
15 \\
4 \\
7 \\
1 \\
1 \\
0 \\
1 \\
1 \\
9 \\
2 \\
2 \\
5\end{array}$ & $\begin{array}{l}0 \\
0 \\
1 \\
0 \\
0 \\
0 \\
0 \\
0 \\
0 \\
0 \\
0 \\
0 \\
0 \\
0 \\
0 \\
0 \\
0 \\
0 \\
0 \\
0\end{array}$ & $\begin{array}{r}9 \\
4 \\
11 \\
11 \\
1 \\
7 \\
3 \\
3 \\
8 \\
2 \\
2 \\
8 \\
4 \\
9 \\
20 \\
8 \\
11 \\
9 \\
16 \\
11 \\
7\end{array}$ & $\begin{array}{l}1 \\
0 \\
0 \\
2 \\
0 \\
1 \\
2 \\
0 \\
1 \\
2 \\
0 \\
0 \\
0 \\
0 \\
0 \\
1 \\
1 \\
1 \\
1 \\
1\end{array}$ & $\begin{array}{l}0 \\
0 \\
0 \\
0 \\
0 \\
0 \\
0 \\
0 \\
0 \\
0 \\
0 \\
0 \\
0 \\
0 \\
0 \\
0 \\
0 \\
0 \\
0 \\
0\end{array}$ & $\begin{array}{l}0 \\
0 \\
0 \\
0 \\
0 \\
0 \\
0 \\
0 \\
0 \\
0 \\
0 \\
0 \\
0 \\
0 \\
0 \\
0 \\
0 \\
0 \\
0 \\
0\end{array}$ & $\begin{array}{l}0 \\
0 \\
0 \\
0 \\
0 \\
0 \\
0 \\
0 \\
0 \\
0 \\
0 \\
0 \\
1 \\
0 \\
0 \\
0 \\
0 \\
0 \\
0 \\
0\end{array}$ & $\begin{array}{l}0 \\
0 \\
0 \\
0 \\
0 \\
0 \\
0 \\
0 \\
0 \\
0 \\
0 \\
0 \\
0 \\
0 \\
0 \\
0 \\
0 \\
0 \\
0 \\
0\end{array}$ & $\begin{array}{r}31 \\
26 \\
15 \\
0 \\
45 \\
9 \\
3 \\
10 \\
1 \\
5 \\
4 \\
23 \\
5 \\
15 \\
12 \\
24 \\
11 \\
8 \\
8 \\
11\end{array}$ & $\begin{array}{r}35 \\
22 \\
21 \\
8 \\
5 \\
24 \\
9 \\
12 \\
5 \\
13 \\
14 \\
12 \\
11 \\
28 \\
15 \\
28 \\
24 \\
24 \\
34 \\
24\end{array}$ & $\begin{array}{l}0 \\
0 \\
0 \\
0 \\
0 \\
0 \\
0 \\
0 \\
0 \\
0 \\
0 \\
0 \\
1 \\
0 \\
0 \\
0 \\
0 \\
0 \\
0 \\
0\end{array}$ & $\begin{array}{l}0 \\
0 \\
0 \\
0 \\
0 \\
0 \\
0 \\
0 \\
0 \\
0 \\
0 \\
0 \\
0 \\
0 \\
0 \\
0 \\
0 \\
0 \\
0 \\
0\end{array}$ & $\begin{array}{l}0 \\
0 \\
0 \\
0 \\
0 \\
0 \\
0 \\
0 \\
0 \\
0 \\
0 \\
0 \\
0 \\
0 \\
0 \\
0 \\
0 \\
0 \\
0 \\
0\end{array}$ & $\begin{array}{l}500 \\
500 \\
500 \\
500 \\
500 \\
500 \\
500 \\
500 \\
500 \\
500 \\
500 \\
500 \\
500 \\
500 \\
500 \\
500 \\
500 \\
500 \\
500 \\
500\end{array}$ & $\begin{array}{l}0 \\
0 \\
0 \\
0 \\
0 \\
0 \\
0 \\
0 \\
0 \\
0 \\
0 \\
0 \\
0 \\
0 \\
0 \\
0 \\
0 \\
0 \\
0 \\
0\end{array}$ & $\begin{array}{l}0 \\
0 \\
0 \\
0 \\
0 \\
0 \\
0 \\
0 \\
0 \\
0 \\
0 \\
0 \\
0 \\
0 \\
0 \\
0 \\
0 \\
0 \\
0 \\
0\end{array}$ & $\begin{array}{l}0 \\
0 \\
0 \\
2 \\
0 \\
0 \\
0 \\
0 \\
2 \\
0 \\
0 \\
0 \\
0 \\
0 \\
0 \\
0 \\
0 \\
0 \\
0 \\
0\end{array}$ & $\begin{array}{l}0 \\
0 \\
0 \\
0 \\
0 \\
0 \\
0 \\
0 \\
0 \\
0 \\
0 \\
0 \\
0 \\
0 \\
0 \\
0 \\
0 \\
0 \\
0 \\
0\end{array}$ & $\begin{array}{l}0 \\
0 \\
0 \\
0 \\
0 \\
0 \\
0 \\
0 \\
0 \\
0 \\
0 \\
0 \\
0 \\
0 \\
0 \\
0 \\
0 \\
0 \\
0 \\
0\end{array}$ \\
\hline $\begin{array}{l}0 \\
0 \\
0 \\
0\end{array}$ & $\begin{array}{l}0 \\
0 \\
0 \\
0\end{array}$ & $\begin{array}{l}0 \\
5 \\
0 \\
3\end{array}$ & $\begin{array}{r}6 \\
20 \\
23 \\
1\end{array}$ & $\begin{array}{l}1 \\
0 \\
0 \\
0\end{array}$ & $\begin{array}{r}1 \\
10 \\
4 \\
5\end{array}$ & $\begin{array}{l}1 \\
2 \\
9 \\
0\end{array}$ & $\begin{array}{l}0 \\
0 \\
0 \\
0\end{array}$ & $\begin{array}{l}0 \\
0 \\
0 \\
0\end{array}$ & $\begin{array}{l}1 \\
0 \\
0 \\
0\end{array}$ & $\begin{array}{l}0 \\
0 \\
0 \\
0\end{array}$ & $\begin{array}{l}1 \\
4 \\
1 \\
8\end{array}$ & $\begin{array}{r}6 \\
20 \\
6 \\
15\end{array}$ & $\begin{array}{l}0 \\
0 \\
0 \\
0\end{array}$ & $\begin{array}{l}1 \\
0 \\
1 \\
0\end{array}$ & $\begin{array}{l}0 \\
0 \\
0 \\
0\end{array}$ & $\begin{array}{l}500 \\
500 \\
500 \\
500\end{array}$ & $\begin{array}{l}7 \\
4 \\
1 \\
3\end{array}$ & $\begin{array}{l}0 \\
0 \\
0 \\
0\end{array}$ & $\begin{array}{l}0 \\
0 \\
0 \\
0\end{array}$ & $\begin{array}{l}0 \\
0 \\
0 \\
0\end{array}$ & \\
\hline $\begin{array}{l}0 \\
0\end{array}$ & $\begin{array}{l}1 \\
0 \\
0 \\
0 \\
0 \\
0 \\
0 \\
0 \\
0 \\
0\end{array}$ & $\begin{array}{l}0 \\
4 \\
2 \\
3 \\
1 \\
0 \\
2 \\
0 \\
1 \\
0\end{array}$ & $\begin{array}{r}18 \\
1 \\
16 \\
2 \\
1 \\
15 \\
3 \\
13 \\
1 \\
1 \\
0\end{array}$ & $\begin{array}{l}0 \\
0 \\
0 \\
0 \\
0 \\
0 \\
0 \\
0 \\
0 \\
0\end{array}$ & $\begin{array}{r}3 \\
7 \\
6 \\
6 \\
4 \\
1 \\
5 \\
18 \\
3 \\
0\end{array}$ & $\begin{array}{r}10 \\
2 \\
13 \\
0 \\
2 \\
6 \\
1 \\
1 \\
1 \\
0 \\
0\end{array}$ & $\begin{array}{l}0 \\
0 \\
0 \\
0 \\
0 \\
0 \\
0 \\
0 \\
0 \\
0\end{array}$ & $\begin{array}{l}0 \\
0 \\
0 \\
0 \\
0 \\
0 \\
0 \\
0 \\
0 \\
0\end{array}$ & $\begin{array}{l}0 \\
0 \\
0 \\
0 \\
0 \\
0 \\
0 \\
0 \\
0 \\
0\end{array}$ & $\begin{array}{l}0 \\
0 \\
0 \\
0 \\
0 \\
0 \\
0 \\
0 \\
0 \\
0\end{array}$ & $\begin{array}{r}5 \\
2 \\
9 \\
19 \\
7 \\
8 \\
1 \\
4 \\
4 \\
5 \\
1\end{array}$ & $\begin{array}{r}8 \\
11 \\
41 \\
33 \\
22 \\
4 \\
6 \\
32 \\
10 \\
2\end{array}$ & $\begin{array}{l}0 \\
0 \\
1 \\
0 \\
0 \\
2 \\
0 \\
1 \\
0 \\
0\end{array}$ & $\begin{array}{l}0 \\
0 \\
0 \\
1 \\
0 \\
0 \\
0 \\
0 \\
0 \\
0\end{array}$ & $\begin{array}{l}0 \\
0 \\
0 \\
0 \\
0 \\
0 \\
0 \\
0 \\
0 \\
0\end{array}$ & $\begin{array}{l}500 \\
500 \\
500 \\
500 \\
500 \\
500 \\
500 \\
500 \\
500 \\
500\end{array}$ & $\begin{array}{r}1 \\
0 \\
0 \\
1 \\
5 \\
3 \\
0 \\
2 \\
10 \\
0\end{array}$ & $\begin{array}{l}0 \\
0 \\
0 \\
0 \\
0 \\
0 \\
0 \\
0 \\
0 \\
0\end{array}$ & $\begin{array}{l}0 \\
0 \\
0 \\
0 \\
0 \\
0 \\
0 \\
0 \\
0 \\
0\end{array}$ & $\begin{array}{l}0 \\
0 \\
0 \\
0 \\
0 \\
0 \\
0 \\
0 \\
0 \\
0\end{array}$ & $\begin{array}{l}0 \\
0 \\
0 \\
0 \\
0 \\
1 \\
0 \\
0 \\
0 \\
0\end{array}$ \\
\hline $\begin{array}{l}2 \\
0 \\
0\end{array}$ & $\begin{array}{l}1 \\
0 \\
0 \\
0 \\
0 \\
0 \\
0 \\
0 \\
2 \\
0 \\
0\end{array}$ & $\begin{array}{l}4 \\
0 \\
1\end{array}$ & $\begin{array}{r}5 \\
8 \\
3 \\
7 \\
71 \\
16 \\
38 \\
23 \\
0 \\
8 \\
3\end{array}$ & $\begin{array}{l}0 \\
0 \\
0 \\
0 \\
0 \\
0 \\
0 \\
0 \\
0 \\
0 \\
0\end{array}$ & $\begin{array}{r}2 \\
15 \\
5 \\
3 \\
13 \\
3 \\
1 \\
4 \\
4 \\
10 \\
0 \\
2\end{array}$ & $\begin{array}{r}1 \\
0 \\
1 \\
6 \\
0 \\
5 \\
6 \\
2 \\
2 \\
15 \\
0 \\
0\end{array}$ & $\begin{array}{l}0 \\
1 \\
0 \\
0 \\
0 \\
0 \\
0 \\
0 \\
0 \\
0 \\
0\end{array}$ & $\begin{array}{l}0 \\
0 \\
0 \\
0 \\
0 \\
0 \\
0 \\
0 \\
0 \\
0 \\
0\end{array}$ & $\begin{array}{l}0 \\
0 \\
0 \\
1 \\
0 \\
0 \\
0 \\
1 \\
0\end{array}$ & $\begin{array}{l}0 \\
0 \\
0 \\
0 \\
0 \\
0 \\
0 \\
0 \\
0 \\
0 \\
1\end{array}$ & $\begin{array}{r}11 \\
3 \\
14 \\
5 \\
9 \\
2 \\
1 \\
16 \\
18 \\
0 \\
0 \\
2\end{array}$ & $\begin{array}{l}16 \\
10 \\
41 \\
20 \\
20 \\
15 \\
20 \\
39 \\
28\end{array}$ & $\begin{array}{l}0 \\
1 \\
0 \\
0 \\
1 \\
0 \\
0 \\
1 \\
1 \\
0 \\
0\end{array}$ & $\begin{array}{l}0 \\
0 \\
0 \\
0 \\
0 \\
0 \\
0 \\
0 \\
1 \\
0 \\
0\end{array}$ & $\begin{array}{l}0 \\
0 \\
0 \\
0 \\
0 \\
0 \\
0 \\
0 \\
0 \\
0 \\
0\end{array}$ & $\begin{array}{l}500 \\
500 \\
500 \\
500 \\
500 \\
500 \\
500 \\
500 \\
500 \\
500 \\
500\end{array}$ & $\begin{array}{r}0 \\
1 \\
0 \\
1 \\
0 \\
1 \\
2 \\
2 \\
0 \\
2 \\
36\end{array}$ & $\begin{array}{l}0 \\
0 \\
0 \\
0 \\
0 \\
0 \\
0 \\
0 \\
0 \\
0 \\
0\end{array}$ & $\begin{array}{l}0 \\
0 \\
0 \\
0 \\
0 \\
0 \\
0 \\
0 \\
0 \\
0 \\
0\end{array}$ & $\begin{array}{l}0 \\
0 \\
0 \\
0 \\
0 \\
0 \\
0 \\
0 \\
0 \\
0 \\
0\end{array}$ & $\begin{array}{l}0 \\
0 \\
0 \\
0 \\
1 \\
0 \\
0 \\
0 \\
0 \\
0 \\
4\end{array}$ \\
\hline $\begin{array}{l}0 \\
1 \\
0 \\
0\end{array}$ & $\begin{array}{l}0 \\
0 \\
0 \\
0 \\
0 \\
0 \\
0 \\
0 \\
0 \\
2 \\
0 \\
0 \\
0 \\
0 \\
0\end{array}$ & $\begin{array}{l}0 \\
2 \\
0\end{array}$ & $\begin{array}{r}5 \\
7 \\
15 \\
2 \\
0 \\
4 \\
1 \\
9 \\
0 \\
9 \\
3 \\
3 \\
01 \\
1 \\
1 \\
3\end{array}$ & $\begin{array}{l}0 \\
0 \\
0 \\
0 \\
0 \\
0 \\
1 \\
0 \\
0 \\
0 \\
0 \\
0\end{array}$ & $\begin{array}{r}2 \\
10 \\
5 \\
3 \\
0 \\
6 \\
6 \\
2 \\
2 \\
3 \\
4 \\
10 \\
8 \\
7 \\
3 \\
0\end{array}$ & $\begin{array}{l}2 \\
6 \\
1 \\
1 \\
0 \\
7 \\
0 \\
0 \\
1 \\
2 \\
2 \\
1 \\
5 \\
2 \\
2\end{array}$ & $\begin{array}{l}0 \\
0 \\
0 \\
0 \\
0 \\
0 \\
0 \\
0 \\
0 \\
0 \\
0 \\
0 \\
0\end{array}$ & $\begin{array}{l}0 \\
0 \\
0 \\
0 \\
0 \\
0 \\
0 \\
0 \\
0 \\
0 \\
0 \\
0 \\
0 \\
0 \\
0\end{array}$ & $\begin{array}{l}0 \\
0 \\
2 \\
0 \\
0 \\
0 \\
0 \\
0 \\
0 \\
0 \\
0 \\
0\end{array}$ & $\begin{array}{l}0 \\
0 \\
0 \\
0 \\
0 \\
0 \\
0 \\
0 \\
0 \\
0 \\
0 \\
0 \\
0\end{array}$ & $\begin{array}{r}6 \\
23 \\
13 \\
3 \\
3 \\
5 \\
27 \\
8 \\
5 \\
5 \\
2 \\
13 \\
5 \\
0 \\
15 \\
10 \\
0\end{array}$ & $\begin{array}{r}11 \\
34 \\
34 \\
7 \\
7 \\
27 \\
10 \\
9 \\
12 \\
24 \\
19 \\
7 \\
65 \\
7 \\
14\end{array}$ & $\begin{array}{l}0 \\
0 \\
0 \\
0 \\
7 \\
0 \\
0 \\
1 \\
0 \\
0 \\
0 \\
0 \\
0 \\
0 \\
0\end{array}$ & $\begin{array}{l}0 \\
0 \\
0 \\
0 \\
0 \\
0 \\
0 \\
0 \\
0 \\
0 \\
0 \\
0 \\
0 \\
0 \\
0\end{array}$ & $\begin{array}{l}0 \\
0 \\
0 \\
0 \\
0 \\
0 \\
0 \\
0 \\
0 \\
0 \\
0 \\
0\end{array}$ & $\begin{array}{l}500 \\
500 \\
500 \\
500 \\
500 \\
500 \\
500 \\
500 \\
500 \\
500 \\
500 \\
500 \\
400 \\
500 \\
500\end{array}$ & $\begin{array}{r}0 \\
7 \\
8 \\
2 \\
3 \\
1 \\
3 \\
7 \\
6 \\
6 \\
13 \\
5 \\
0 \\
0\end{array}$ & $\begin{array}{l}0 \\
0 \\
1 \\
0 \\
0 \\
0 \\
0 \\
1 \\
1 \\
0 \\
0 \\
0\end{array}$ & $\begin{array}{l}0 \\
0 \\
0 \\
0 \\
0 \\
0 \\
0 \\
0 \\
0 \\
0 \\
0 \\
0 \\
0 \\
0 \\
0\end{array}$ & $\begin{array}{l}0 \\
0 \\
0 \\
0 \\
0 \\
0 \\
0 \\
0 \\
0 \\
0 \\
0 \\
0 \\
0 \\
0 \\
0\end{array}$ & $\begin{array}{l}0 \\
2 \\
1 \\
0 \\
0 \\
0 \\
1 \\
0 \\
1 \\
0 \\
0 \\
0 \\
0 \\
0 \\
0\end{array}$ \\
\hline
\end{tabular}

in felsitic volcanic lithic fragments. These differences could be attributed to a higher felsic volcanic input in the Sumisu Rift than in the forearc sites. The framework-grain percent pumice + other colorless glass is higher in samples from Sites 788 (average 58\%), 790 (average $44 \%$ ), and 791 (average 56\%) than from forearc Sites 787 (average $8 \%$ ), 792 (average 15\%), and 793 (average $31 \%$ ). These differences probably result from wind and ocean-current dispersal patterns that favor backarc distribution of the pumiceous and colorless glass fragments. The relative enrichment of colorless lathwork fragments (Fig. 5) in sands from Site 788 could be a function of proximity to the source volcanoes.
Proportions of colorless, brown, and black glassy volcanic lithic grains within samples and among sites are highly variable (Table 3; Plate 1, Figs. 2 and 4; Plate 3, Figs. 3 and 4; Plate 4, Fig. 1). Many samples from backarc Sites 790 and 791 are enriched with respect to brown and black glass in comparison with Site 788 on the rift flank, which has more colorless glass (Fig. 6). Sites 792 and 793 in the forearc basin are much more variable in composition, with significantly less colorless glass lithics than the backarc and sites (Fig. 7). Site 787 is clearly enriched in black glass, and it shows the most limited range in composition of all the Leg 126 sites (Fig. 7). The inhomogeneity of glass color within the Leg 126 samples could imply 
Table 2 (continued).

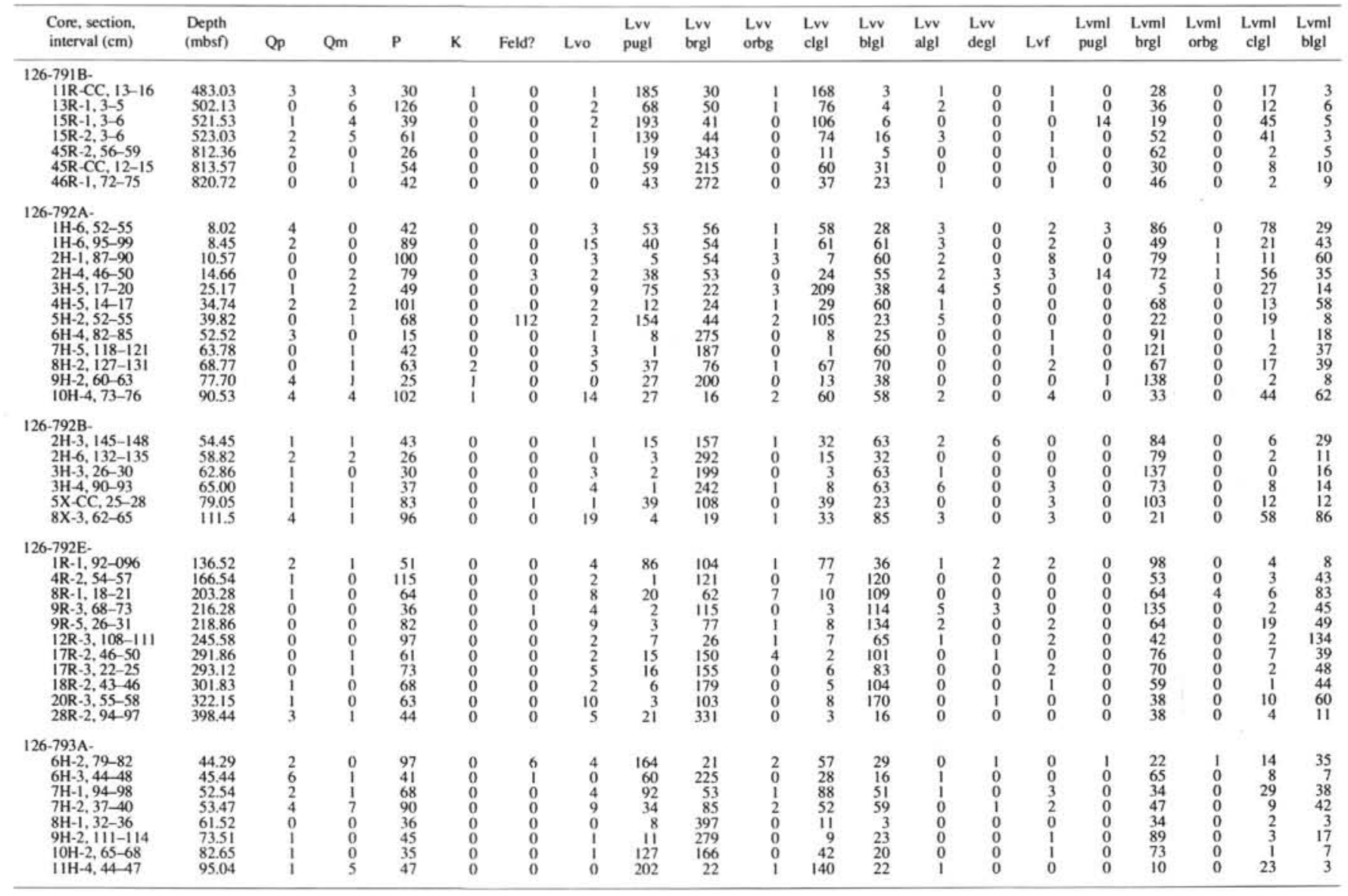

either a physical mixing of pyroclastic debris from different eruptions of varying composition or mixing caused by variations in eruption products at a single volcano. Trends in textures among variously colored glasses show that the majority of colorless glassy fragments are vitric lithic fragments, whereas the brown and black glassy fragments show similar ranges of vitric, microlitic, and lathwork textures (Fig. 8). Therefore, the brown and black glasses may be compositionally similar but differ only in their cooling rates, as discussed above.

Volcanic lithic proportions at Site 787 are distinguished by their high proportion of crystal-rich ( $\mathrm{Lvml}$ and $\mathrm{Lvl}$ ) volcanic lithic fragments (Fig. 8) and higher percentage of black glassy lithics overall (Fig. 7). This site is located along the axis of a submarine canyon that crosses the Izu-Bonin forearc basin north of Aoga Shima (Fig. 2) and heads near the Island of Hachijo Jima, a large emergent volcano (north of Fig. 2). The high concentration of denser tachylitic and crystal-rich grains suggests that the winnowing effects of currents moving down this channel may have preferentially removed lighter glassy fragments, concentrating the denser fragments. Alternatively, the anoma- lous but uniform composition of these sands might reflect a different source region, near Hachijo Island. The high percentage of black glassy fragments at Site 787 (Fig. 7) could be a compositional signature of an island point source of sediment where large volumes of tachylite grains may have been produced either during subaerial mafic eruptions or by weathering of subaerial flows.

\section{TEMPORAL TRENDS}

There are no significant differences between Quaternary and Pliocene sands at Site 788 (Table 4), but at Site 792 there is a trend toward less colorless glass with greater age (Fig. 9). Miocene sands are dominated by brown and black glass. This may be an indication of arc evolution through the Cenozoic from more mafic toward more felsic compositions. Alternatively, this trend might be a function of increased colorless glass dissolution/alteration through time (see Marsaglia and Tazaki, this volume).

Initially, it was hoped that the detrital modes of these volcaniclastic sands might be helpful in verifying shipboard lithologic correlations 
Table 2 (continued).

\begin{tabular}{|c|c|c|c|c|c|c|c|c|c|c|c|c|c|c|c|c|c|c|c|c|c|}
\hline $\begin{array}{l}\text { Lvml } \\
\text { algl }\end{array}$ & $\begin{array}{l}\text { Lvml } \\
\text { degl }\end{array}$ & $\begin{array}{l}\text { Lv1 } \\
\text { pugl }\end{array}$ & $\begin{array}{l}\text { Lvl } \\
\text { brgl }\end{array}$ & $\begin{array}{l}\text { Lv1 } \\
\text { orbg }\end{array}$ & $\begin{array}{l}\text { Lvl } \\
\text { clgl }\end{array}$ & $\begin{array}{l}\text { Lvl } \\
\text { blg1 }\end{array}$ & $\begin{array}{l}\text { Lv1 } \\
\text { algl }\end{array}$ & $\begin{array}{l}\text { Lvl } \\
\text { degl }\end{array}$ & LmLs & B & $\underset{\text { opaq }}{\text { D }}$ & $\begin{array}{c}\text { D } \\
\text { ol/py }\end{array}$ & $\begin{array}{c}\text { D } \\
\text { epid }\end{array}$ & Cement? & Glauc & $\begin{array}{c}\text { Total } \\
\text { pts }\end{array}$ & $\begin{array}{l}\text { Bio } \\
\text { for }\end{array}$ & $\begin{array}{l}\text { Bio } \\
\text { di/ra }\end{array}$ & $\begin{array}{l}\text { Bio } \\
\text { spic }\end{array}$ & $\begin{array}{l}\text { Bio } \\
\text { alg }\end{array}$ & $\begin{array}{l}\text { Bio } \\
\text { other }\end{array}$ \\
\hline 0 & 0 & 2 & 5 & 0 & 4 & 3 & 0 & 0 & 0 & 0 & 1 & & 0 & 1 & 0 & 500 & 0 & 0 & 1 & 0 & 1 \\
\hline 0 & 0 & 0 & 13 & 0 & 7 & 2 & 0 & 0 & 0 & 0 & 36 & 50 & 1 & 1 & 0 & 500 & 3 & 0 & 0 & 0 & 0 \\
\hline 0 & 0 & 4 & 6 & 0 & 4 & 1 & 0 & 0 & 0 & 0 & $\begin{array}{l}30 \\
0\end{array}$ & 10 & 0 & 0 & 0 & 500 & 0 & 0 & 0 & 0 & i \\
\hline 0 & 0 & 0 & II & 0 & 17 & 5 & 0 & 0 & 0 & 0 & 10 & is & 0 & 0 & 0 & 500 & I & i & 0 & 0 & 0 \\
\hline 0 & 0 & 0 & 4 & 0 & 0 & I & 0 & 0 & 0 & 0 & 3 & is & 0 & 0 & 0 & 500 & 10 & 0 & 0 & 0 & 0 \\
\hline 0 & $\begin{array}{l}0 \\
0\end{array}$ & 0 & 19 & 0 & 1 & 7 & 1 & 0 & 0 & 0 & 1 & 3 & 0 & 0 & 0 & 500 & 62 & 0 & 0 & 0 & 0 \\
\hline 0 & & 0 & 16 & 0 & 0 & 6 & 0 & 0 & 0 & 0 & 0 & 2 & 0 & 0 & 0 & 500 & 25 & 1 & 0 & 0 & 0 \\
\hline 0 & 0 & 2 & 20 & 0 & 9 & 3 & 0 & 0 & 0 & 0 & 2 & 16 & 1 & 1 & 0 & 500 & 8 & 0 & 1 & 0 & I \\
\hline 2 & 0 & 0 & 5 & 0 & 4 & 12 & 0 & 0 & i & 0 & 8 & 26 & 0 & 0 & 0 & 500 & 2 & 0 & 0 & 0 & 0 \\
\hline${ }_{0}^{0}$ & 0 & 0 & 11 & 0 & 4 & 18 & 0 & 0 & 0 & 0 & 20 & 54 & 0 & 0 & 0 & 500 & 5 & 0 & 0 & 0 & 0 \\
\hline $\begin{array}{l}0 \\
0\end{array}$ & ${ }_{0}^{0}$ & 1 & 6 & 0 & 5 & 6 & 0 & 0 & 0 & 0 & 6 & 33 & I & 0 & 0 & 500 & 1 & ${ }_{0}^{0}$ & 0 & 0 & 0 \\
\hline 0 & $\begin{array}{l}0 \\
0\end{array}$ & 0 & 15 & 0 & 8 & $14^{2}$ & $\begin{array}{l}0 \\
0\end{array}$ & $\begin{array}{l}0 \\
0 \\
0\end{array}$ & ${ }_{0}^{2}$ & $\begin{array}{l}0 \\
0\end{array}$ & $31^{5}$ & $\begin{array}{l}20 \\
59\end{array}$ & ${ }_{0}^{2}$ & $\begin{array}{l}0 \\
0\end{array}$ & $\begin{array}{l}0 \\
0\end{array}$ & $\begin{array}{l}500 \\
500\end{array}$ & 8 & $\begin{array}{l}0 \\
0\end{array}$ & $\begin{array}{l}1 \\
0\end{array}$ & $\begin{array}{l}0 \\
0\end{array}$ & 0 \\
\hline 0 & 0 & 0 & 4 & 1 & 5 & 6 & 0 & 0 & 2 & 0 & 2 & 15 & 0 & 0 & 0 & 500 & 1 & 0 & 0 & 0 & 0 \\
\hline 0 & 0 & 0 & 35 & 0 & 0 & 3 & 0 & 0 & 0 & 0 & 8 & 8 & 0 & 0 & 0 & 500 & 6 & 0 & 0 & 0 & 0 \\
\hline 0 & 0 & 0 & 21 & 0 & 0 & 6 & 0 & 0 & 0 & 0 & 2 & 15 & 0 & 0 & 0 & 500 & 2 & 0 & 0 & 0 & 0 \\
\hline $\begin{array}{l}0 \\
0\end{array}$ & $\begin{array}{l}0 \\
0 \\
0\end{array}$ & $\begin{array}{l}0 \\
0\end{array}$ & $\begin{array}{r}7 \\
18\end{array}$ & $\begin{array}{l}0 \\
0\end{array}$ & $\begin{array}{l}1 \\
2\end{array}$ & 12 & $\begin{array}{l}0 \\
0\end{array}$ & $\begin{array}{l}0 \\
0\end{array}$ & $\begin{array}{l}1 \\
0 \\
0\end{array}$ & $\begin{array}{l}0 \\
0\end{array}$ & 10 & 20 & ${ }_{0}^{2}$ & $\begin{array}{l}0 \\
0 \\
0\end{array}$ & $\begin{array}{l}0 \\
0 \\
0\end{array}$ & 500 & $\begin{array}{r}99 \\
16\end{array}$ & $\begin{array}{l}0 \\
0\end{array}$ & ${ }_{0}^{0}$ & ${ }_{0}^{0}$ & 30 \\
\hline 0 & 0 & 0 & $\begin{array}{l}18 \\
3\end{array}$ & 0 & 4 & 18 & $\begin{array}{l}0 \\
0\end{array}$ & $\begin{array}{l}0 \\
0\end{array}$ & 2 & $\begin{array}{l}0 \\
0\end{array}$ & 5 & 35 & 0 & $\begin{array}{l}0 \\
0\end{array}$ & 0 & 500 & 11 & 0 & $\begin{array}{l}0 \\
0\end{array}$ & 0 & 0 \\
\hline 1 & 0 & 0 & 23 & 0 & 2 & 10 & 0 & 0 & 0 & 0 & 0 & 23 & 0 & 0 & 0 & 500 & 10 & 0 & 0 & 0 & 1 \\
\hline 0 & $\begin{array}{l}0 \\
0\end{array}$ & $\begin{array}{l}0 \\
0\end{array}$ & 21 & 0 & 0 & 2 & $\begin{array}{l}0 \\
0\end{array}$ & $\begin{array}{l}0 \\
0\end{array}$ & $\begin{array}{l}0 \\
0\end{array}$ & $\begin{array}{l}0 \\
0\end{array}$ & $\begin{array}{l}0 \\
3\end{array}$ & $\begin{array}{l}23 \\
10\end{array}$ & $\begin{array}{l}0 \\
0\end{array}$ & $\begin{array}{l}0 \\
0\end{array}$ & $\begin{array}{l}0 \\
0\end{array}$ & $\begin{array}{l}500 \\
500\end{array}$ & $\begin{array}{r}100 \\
5\end{array}$ & 0 & $\begin{array}{l}0 \\
0\end{array}$ & 0 & 0 \\
\hline 1 & 0 & 0 & 27 & 0 & 0 & 5 & 0 & 0 & 0 & 0 & 3 & 9 & 0 & 0 & 0 & 500 & 9 & 1 & i & 0 & 0 \\
\hline 0 & 0 & 0 & 13 & 0 & 1 & 4 & 0 & 0 & 0 & 0 & 5 & 15 & 0 & 0 & 0 & 500 & 0 & 0 & 0 & 0 & 0 \\
\hline${ }_{0}^{3}$ & 2 & 0 & 16 & 0 & 3 & 3 & 0 & 0 & 0 & 0 & 7 & 40 & 0 & 0 & 0 & 500 & 4 & 0 & 0 & 0 & 0 \\
\hline 0 & 0 & 0 & 8 & 0 & 3 & 28 & 0 & 0 & 0 & 0 & 9 & 19 & 0 & 0 & 0 & 500 & 0 & 0 & 0 & 0 & 0 \\
\hline 0 & 0 & 0 & 4 & 0 & 3 & 5 & 0 & 0 & 0 & 0 & 1 & 10 & 0 & 0 & 0 & 500 & 12 & 1 & 2 & 0 & 2 \\
\hline 0 & 0 & 0 & 5 & 0 & 0 & 7 & 0 & 0 & 0 & 0 & 2 & 20 & 0 & 0 & 0 & 30 & 0 & & 0 & & 0 \\
\hline${ }_{0}^{0}$ & 0 & 0 & 6 & 0 & 0 & 17 & 0 & 0 & 0 & 0 & 2 & 35 & 0 & 2 & 0 & 500 & 1 & 1 & 1 & 0 & 0 \\
\hline $\begin{array}{l}0 \\
0 \\
0\end{array}$ & $\begin{array}{l}0 \\
0 \\
0\end{array}$ & $\begin{array}{l}0 \\
0\end{array}$ & 10 & ${ }_{0}^{0}$ & 1 & ${ }^{8}$ & $\begin{array}{l}0 \\
0\end{array}$ & 0 & 0 & 0 & 2 & 13 & ${ }_{0}^{0}$ & 1 & ${ }_{0}^{0}$ & 500 & ${ }_{0}^{0}$ & ${ }_{0}^{0}$ & ${ }_{0}^{0}$ & $\begin{array}{l}0 \\
0 \\
0\end{array}$ & 0 \\
\hline $\begin{array}{l}0 \\
0\end{array}$ & $\begin{array}{l}0 \\
0\end{array}$ & $\begin{array}{l}0 \\
0\end{array}$ & 11 & 0 & 0 & $\begin{array}{l}13 \\
33\end{array}$ & $\begin{array}{l}0 \\
0 \\
0\end{array}$ & $\begin{array}{l}0 \\
0\end{array}$ & $\begin{array}{l}0 \\
0\end{array}$ & $\begin{array}{l}0 \\
0\end{array}$ & 0 & $\begin{array}{l}29 \\
69\end{array}$ & $\begin{array}{l}0 \\
0\end{array}$ & $\begin{array}{l}0 \\
0 \\
0\end{array}$ & $\begin{array}{l}0 \\
0 \\
0\end{array}$ & 500 & $\frac{0}{7}$ & $\begin{array}{l}0 \\
0\end{array}$ & 0 & $\begin{array}{l}0 \\
0\end{array}$ & $\begin{array}{l}0 \\
0\end{array}$ \\
\hline i & 0 & 0 & 8 & 0 & 3 & 13 & 0 & 0 & 0 & 0 & 3 & 11 & 0 & 0 & 2 & 50 & 2 & 0 & 0 & 0 & 1 \\
\hline 0 & 0 & 0 & 13 & 0 & 0 & 5 & 0 & 0 & i & 0 & 5 & is & 0 & 0 & 0 & 500 & 4 & 2 & 0 & 0 & i \\
\hline $\begin{array}{l}0 \\
0\end{array}$ & $\begin{array}{l}0 \\
0\end{array}$ & ${ }_{0}^{0}$ & 4 & ${ }_{0}^{0}$ & $\begin{array}{l}0 \\
0\end{array}$ & 11 & 0 & 0 & 0 & 0 & 2 & 13 & 0 & $\begin{array}{l}0 \\
0 \\
0\end{array}$ & 0 & 500 & 1 & 13 & ${ }_{0}^{0}$ & ${ }_{0}^{0}$ & 0 \\
\hline 1 & $\begin{array}{l}0 \\
0\end{array}$ & $\begin{array}{l}0 \\
0\end{array}$ & $\frac{3}{7}$ & 0 & $\begin{array}{l}0 \\
0\end{array}$ & $\frac{9}{2}$ & $\begin{array}{l}0 \\
0\end{array}$ & $\begin{array}{l}0 \\
0 \\
0\end{array}$ & $\begin{array}{l}0 \\
0\end{array}$ & $\begin{array}{l}0 \\
0\end{array}$ & $\begin{array}{l}1 \\
0\end{array}$ & $\begin{array}{l}18 \\
13\end{array}$ & $\begin{array}{l}0 \\
0\end{array}$ & $\begin{array}{l}0 \\
0 \\
0\end{array}$ & $\begin{array}{l}0 \\
0\end{array}$ & $\begin{array}{l}500 \\
5\end{array}$ & $\begin{array}{l}8 \\
7\end{array}$ & ${ }^{6}$ & $\begin{array}{l}0 \\
0\end{array}$ & $\begin{array}{l}0 \\
0\end{array}$ & $\begin{array}{l}0 \\
0\end{array}$ \\
\hline 0 & 0 & 1 & 1 & 1 & 7 & 2 & 0 & 0 & 1 & 0 & 5 & 25 & I & 0 & 0 & 50 & 55 & 0 & 0 & 0 & 0 \\
\hline 0 & 0 & 0 & 12 & 0 & 2 & 4 & 0 & 0 & 0 & 0 & 2 & 20 & $\mathrm{I}$ & 0 & 0 & 50 & 9 & 0 & 0 & 0 & i \\
\hline 0 & 0 & i & 7 & 0 & 5 & 6 & 0 & 0 & 0 & 0 & 0 & 16 & 0 & 0 & 0 & 50 & 19 & 0 & 0 & 0 & 0 \\
\hline 0 & 0 & 0 & 9 & 0 & 2 & 19 & 0 & 0 & 0 & 0 & 3 & 23 & i & 0 & 0 & 50 & 22 & 0 & 0 & 0 & 0 \\
\hline 0 & 0 & 0 & 2 & 0 & 0 & 0 & 0 & 0 & 0 & 0 & 1 & 3 & 0 & 0 & 0 & 50 & 7 & 0 & 0 & 0 & 0 \\
\hline $\begin{array}{l}0 \\
0\end{array}$ & $\begin{array}{l}0 \\
0 \\
0\end{array}$ & $\begin{array}{l}0 \\
0\end{array}$ & 7 & ${ }_{0}^{0}$ & 0 & 1 & 0 & $\begin{array}{l}0 \\
0\end{array}$ & ${ }_{0}^{0}$ & 0 & $\frac{2}{3}$ & 11 & 0 & ${ }_{0}^{0}$ & ${ }_{0}^{0}$ & 50 & 3 & ${ }_{0}^{0}$ & 0 & $\begin{array}{l}0 \\
0 \\
0\end{array}$ & $\begin{array}{l}0 \\
0\end{array}$ \\
\hline $\begin{array}{l}0 \\
0\end{array}$ & $\begin{array}{l}0 \\
0\end{array}$ & $\begin{array}{l}0 \\
0\end{array}$ & $\begin{array}{l}8 \\
3\end{array}$ & 0 & 4 & 1 & $\begin{array}{l}0 \\
0\end{array}$ & $\begin{array}{l}0 \\
0\end{array}$ & $\begin{array}{l}0 \\
1\end{array}$ & $\begin{array}{l}0 \\
0\end{array}$ & 1 & $\begin{array}{l}13 \\
13\end{array}$ & $\begin{array}{l}0 \\
0\end{array}$ & $\begin{array}{l}0 \\
0\end{array}$ & $\begin{array}{l}0 \\
0\end{array}$ & $\begin{array}{l}500 \\
500\end{array}$ & 3 & 0 & 0 & $\begin{array}{l}0 \\
0\end{array}$ & $\begin{array}{l}0 \\
0\end{array}$ \\
\hline
\end{tabular}

between Sites 790 and 791 . However, plots of recalculated parameters vs. depth do not show correlatable trends between sites. This lack of correlation between Sites 790 and 791 may be a function of the sample spacing used in this study (an average of one per sand-bearing core) in conjunction with variable recovery rates throughout the cored intervals.

Within the sections at Sites 790 and 791, the felsic volcanic component (total percent gravel through silt) increases upsection (Taylor, Fujioka, et al., 1990, pp. 127-220). The sand fractions examined in this study do not show a temporal increase in colorless components; instead, sand compositions fluctuate between colorless and brown glass (Fig. 6) with a higher proportion of black glass (tachylite) at Site 790. The likely sources of the felsic pumice fragments are arc eruptions, whereas the more mafic component is probably derived from intrarift mafic volcanic centers, which may have been sources for "hyaloclastite-induced submarine density flows" (Taylor, Fujioka, et al., 1990, pp. 127-220).

\section{COMPARISON WITH MARIANA TRANSECT SITES}

Packer and Ingersoll (1986) present sand and sandstone detrital modes for 60 samples from 7 sites on a transect across the Mariana forearc, arc axis, and Mariana Trough, an actively spreading backarc basin (Fig. 1). Their ranges of QFL compositions are similar to those found in the Izu-Bonin transect (Fig. 10). In general, Leg 126 samples are slightly enriched in quartz as compared with the Leg 59 and 60 samples analyzed by Packer and Ingersoll (1986). The slight compositional difference between the arc segments could be a function of more silicic volcanism to the north in the vicinity of the Sumisu Rift.

Unfortunately, Packer and Ingersoll (1986) did not differentiate volcanic sand on the basis of color, only on texture. However, two site reports from Leg 60 (Shipboard Scientific Party, 1981a, 1981b) describe glass colors in recovered cores (e.g., colorless, pale yellow, pale green, pale brown, and brown) similar to the range seen in the Leg 126 samples. Similar to Leg 126 volcanic sands, Leg 60 samples have diverse shard compositions (colors) for any given sample, with one glass type usually predominant (Schmincke, 1981). Schmincke (1981) attributed this heterogeneity to mixing of ashes during sediment transport by turbidity currents. A downhole increase in brown glass was observed at Site 453 , which was attributed to alteration (Shipboard Scientific Party, 1981a).

\section{SUMMARY AND CONCLUSIONS}

Modal compositions of volcaniclastic sands recovered on Leg 126 (Izu-Bonin island arc and Sumisu Rift) are similar to those from the Mariana magmatic-arc system. Sand derived from these two intrao ceanic magmatic arcs is characterized by very low quartz $(\mathrm{QFL} \% \mathrm{Q}<3 \%)$, high volcanic lithic (QFL\%L > 60\%), and moderate plagioclase feldspar content $(\mathrm{QFL} \% \mathrm{~F}<39 \%)$. The glass color of volcanic fragments ranges from black (tachylite) to brown to colorless, and individual samples 
Table 3. Recalculated parameters.

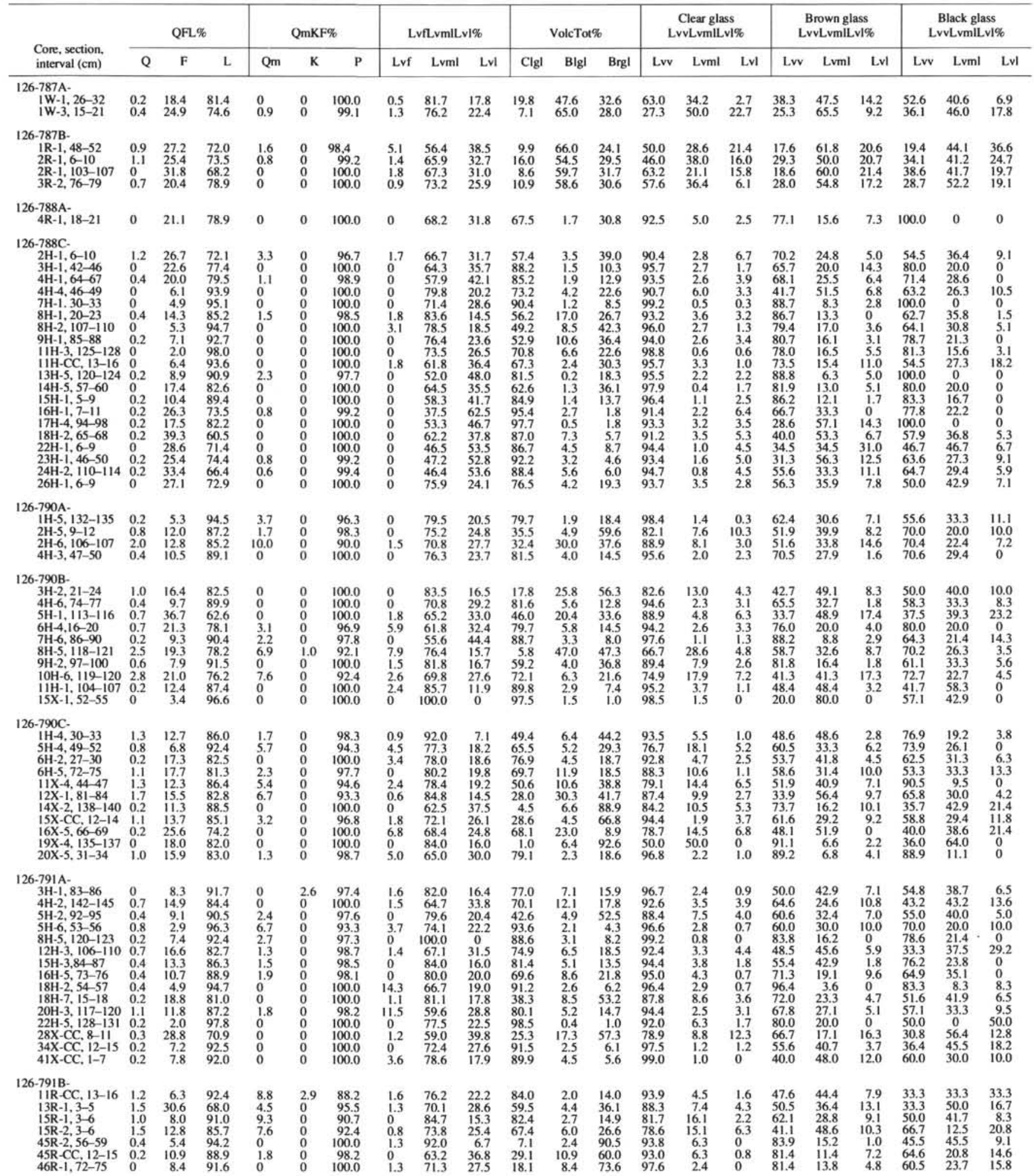


Table 3 (continued).

\begin{tabular}{|c|c|c|c|c|c|c|c|c|c|c|c|c|c|c|c|c|c|c|c|c|c|}
\hline \multirow{2}{*}{$\begin{array}{l}\text { Core, section, } \\
\text { interval }(\mathrm{cm})\end{array}$} & \multicolumn{3}{|c|}{ QFL\% } & \multicolumn{3}{|c|}{ QmKF\% } & \multicolumn{3}{|c|}{ LvflumlLvi\% } & \multicolumn{3}{|c|}{ VolcTot\% } & \multicolumn{3}{|c|}{$\begin{array}{c}\text { Clear glass } \\
\text { LvvLLvilLv1\% }\end{array}$} & \multicolumn{3}{|c|}{$\begin{array}{l}\text { Brown glass } \\
\text { LvvLvmlLv|\% }\end{array}$} & \multicolumn{3}{|c|}{$\begin{array}{c}\text { Black glass } \\
\text { LvvLvmiLvi\% }\end{array}$} \\
\hline & Q & $\mathrm{F}$ & L & $\mathrm{Qm}$ & $\mathrm{K}$ & $\mathbf{P}$ & Lvf & Lvml & Lvl & Clgl & Blgl & Brgl & Lvv & Lvml & Lvl & Lvv & Lvml & LvI & Lvv & Lvml & Lvl \\
\hline $\begin{array}{l}26-792 \mathrm{~A}- \\
1 \mathrm{H}-6,52-55 \\
1 \mathrm{H}-6,95-99 \\
2 \mathrm{H}-1,87-90 \\
2 \mathrm{H}-4,46-50 \\
3 \mathrm{H}-5,17-20 \\
4 \mathrm{H}-5,14-17 \\
5 \mathrm{H}-2,52-55 \\
6 \mathrm{H}-4,82-85 \\
7 \mathrm{H}-5,118-121 \\
8 \mathrm{H}-2,127-131 \\
9 \mathrm{H}-2,60-63 \\
10 \mathrm{H}-4,73-76\end{array}$ & $\begin{array}{l}0.8 \\
0.4 \\
0 \\
0.4 \\
0.6 \\
1.0 \\
0.2 \\
0.6 \\
0.2 \\
0.2 \\
1.0 \\
1.7\end{array}$ & $\begin{array}{r}8.7 \\
19.1 \\
23.5 \\
17.8 \\
10.4 \\
24.6 \\
16.6 \\
3.1 \\
8.7 \\
13.9 \\
5.4 \\
22.4\end{array}$ & $\begin{array}{l}90.4 \\
80.5 \\
76.5 \\
81.7 \\
89.0 \\
74.4 \\
83.2 \\
96.3 \\
91.1 \\
85.9 \\
93.6 \\
75.9\end{array}$ & $\begin{array}{l}0 \\
0 \\
0 \\
2.5 \\
3.9 \\
1.9 \\
1.4 \\
0 \\
2.3 \\
1.5 \\
3.7 \\
3.7\end{array}$ & $\begin{array}{l}0 \\
0 \\
0 \\
0 \\
0 \\
0 \\
0 \\
0 \\
0 \\
3.0 \\
3.7 \\
0.9\end{array}$ & $\begin{array}{r}100.0 \\
100.0 \\
100.0 \\
97.5 \\
96.1 \\
98.1 \\
98.6 \\
100.0 \\
97.7 \\
99.5 \\
92.6 \\
95.3\end{array}$ & $\begin{array}{l}0.9 \\
1.4 \\
4.2 \\
1.5 \\
0 \\
0 \\
0 \\
0.7 \\
0.5 \\
1.4 \\
0 \\
2.4\end{array}$ & $\begin{array}{l}84.5 \\
83.5 \\
78.6 \\
89.4 \\
85.2 \\
79.0 \\
75.4 \\
73.8 \\
85.1 \\
84.8 \\
86.6 \\
82.7\end{array}$ & $\begin{array}{l}14.6 \\
15.1 \\
17.2 \\
9.0 \\
14.8 \\
21.0 \\
24.6 \\
25.5 \\
14.4 \\
13.8 \\
13.4 \\
14.9\end{array}$ & $\begin{array}{r}47.7 \\
36.0 \\
87.7 \\
37.8 \\
79.4 \\
20.6 \\
72.6 \\
3.7 \\
0.9 \\
31.0 \\
10.0 \\
41.5\end{array}$ & $\begin{array}{r}14.1 \\
33.1 \\
44.7 \\
26.3 \\
13.6 \\
43.9 \\
9.5 \\
9.9 \\
23.6 \\
30.8 \\
10.9 \\
42.5\end{array}$ & $\begin{array}{l}38.3 \\
30.9 \\
46.6 \\
35.9 \\
7.0 \\
35.5 \\
17.9 \\
86.4 \\
75.5 \\
38.2 \\
79.1 \\
16.0\end{array}$ & $\begin{array}{l}54.7 \\
80.2 \\
44.4 \\
44.9 \\
89.9 \\
66.1 \\
91.5 \\
94.1 \\
50.0 \\
85.2 \\
88.9 \\
64.4\end{array}$ & $\begin{array}{r}39.9 \\
16.7 \\
40.7 \\
50.7 \\
8.5 \\
21.0 \\
6.7 \\
5.9 \\
50.0 \\
13.9 \\
6.7 \\
32.6\end{array}$ & $\begin{array}{r}5.4 \\
3.2 \\
14.8 \\
4.3 \\
1.6 \\
12.9 \\
1.8 \\
0 \\
0 \\
0.8 \\
4.4 \\
3.0\end{array}$ & $\begin{array}{l}34.4 \\
50.0 \\
37.5 \\
40.5 \\
78.6 \\
22.4 \\
62.9 \\
68.6 \\
56.8 \\
50.7 \\
56.2 \\
30.8\end{array}$ & $\begin{array}{l}53.4 \\
45.4 \\
54.9 \\
55.0 \\
17.9 \\
63.6 \\
31.4 \\
22.7 \\
36.8 \\
44.7 \\
38.8 \\
63.5\end{array}$ & $\begin{array}{r}12.3 \\
4.6 \\
7.6 \\
4.6 \\
3.6 \\
14.0 \\
5.7 \\
8.7 \\
6.4 \\
4.7 \\
5.1 \\
5.8\end{array}$ & $\begin{array}{l}46.7 \\
52.6 \\
43.5 \\
57.3 \\
70.4 \\
45.5 \\
62.2 \\
54.3 \\
58.3 \\
57.9 \\
77.6 \\
42.0\end{array}$ & $\begin{array}{l}48.3 \\
37.1 \\
43.5 \\
36.5 \\
25.9 \\
43.9 \\
21.6 \\
39.1 \\
35.9 \\
32.2 \\
16.3 \\
44.9\end{array}$ & $\begin{array}{r}5.0 \\
10.3 \\
13.0 \\
6.3 \\
3.7 \\
10.6 \\
16.2 \\
6.5 \\
5.8 \\
9.9 \\
6.1 \\
13.0\end{array}$ \\
\hline $\begin{array}{l}\text { 126-792B- } \\
2 \mathrm{H}-3,145-148 \\
2 \mathrm{H}-6,132-135 \\
3 \mathrm{H}-3,26-30 \\
3 \mathrm{H}-4,90-93 \\
5 \mathrm{X}-\mathrm{CC}, 25-28 \\
8 \mathrm{X}-3,62-65\end{array}$ & $\begin{array}{l}0.4 \\
0.8 \\
0.2 \\
0.4 \\
0.4 \\
1.1\end{array}$ & $\begin{array}{r}9.0 \\
5.3 \\
6.1 \\
7.7 \\
18.5 \\
20.3\end{array}$ & $\begin{array}{l}90.6 \\
93.8 \\
93.6 \\
91.9 \\
81.0 \\
78.6\end{array}$ & $\begin{array}{l}2.3 \\
7.1 \\
0 \\
2.6 \\
1.2 \\
1.0\end{array}$ & $\begin{array}{l}0 \\
0 \\
0 \\
0 \\
0 \\
0\end{array}$ & $\begin{array}{r}97.7 \\
92.9 \\
100.0 \\
97.4 \\
98.8 \\
99.0\end{array}$ & $\begin{array}{l}0 \\
0 \\
0 \\
2.6 \\
1.9 \\
1.4\end{array}$ & $\begin{array}{l}77.4 \\
80.0 \\
82.8 \\
81.9 \\
84.1 \\
79.7\end{array}$ & $\begin{array}{l}22.6 \\
20.0 \\
17.2 \\
15.5 \\
14.0 \\
18.8\end{array}$ & $\begin{array}{r}13.1 \\
4.4 \\
1.1 \\
4.2 \\
26.0 \\
28.4\end{array}$ & $\begin{array}{r}24.2 \\
9.8 \\
18.6 \\
19.0 \\
10.6 \\
57.7\end{array}$ & $\begin{array}{l}62.7 \\
85.8 \\
80.3 \\
76.8 \\
63.4 \\
13.9\end{array}$ & $\begin{array}{r}85.5 \\
90.0 \\
100.0 \\
50.0 \\
83.9 \\
37.8\end{array}$ & $\begin{array}{c}10.9 \\
10.0 \\
0 \\
44.4 \\
12.9 \\
59.2\end{array}$ & $\begin{array}{l}3.6 \\
0 \\
0 \\
5.6 \\
3.2 \\
3.1\end{array}$ & $\begin{array}{l}59.5 \\
74.5 \\
54.8 \\
73.8 \\
47.6 \\
39.6\end{array}$ & $\begin{array}{l}31.8 \\
20.2 \\
37.7 \\
22.3 \\
45.4 \\
43.8\end{array}$ & $\begin{array}{r}8.7 \\
5.4 \\
7.4 \\
4.0 \\
7.0 \\
16.7\end{array}$ & $\begin{array}{l}61.8 \\
71.1 \\
75.0 \\
77.8 \\
60.5 \\
42.7\end{array}$ & $\begin{array}{l}28.4 \\
24.4 \\
19.0 \\
17.3 \\
31.6 \\
43.2\end{array}$ & $\begin{array}{r}9.8 \\
4.4 \\
6.0 \\
4.9 \\
7.9 \\
14.1\end{array}$ \\
\hline 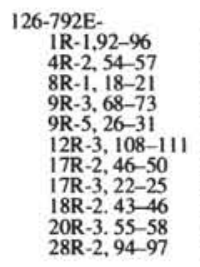 & $\begin{array}{l}0.6 \\
0.2 \\
0.2 \\
0 \\
0 \\
0 \\
0.2 \\
0.2 \\
0.2 \\
0.2 \\
0.8\end{array}$ & $\begin{array}{r}10.4 \\
24.1 \\
13.8 \\
7.6 \\
17.4 \\
22.6 \\
12.6 \\
15.2 \\
14.0 \\
13.1 \\
9.0\end{array}$ & $\begin{array}{r}89.0 \\
75.7 \\
86.0 \\
92.4 \\
82.6 \\
77.4 \\
87.2 \\
84.6 \\
85.8 \\
86.7 \\
90.1\end{array}$ & $\begin{array}{l}1.9 \\
0 \\
0 \\
0 \\
0 \\
0 \\
1.6 \\
1.4 \\
0 \\
0 \\
2.2\end{array}$ & $\begin{array}{l}0 \\
0 \\
0 \\
0 \\
0 \\
0 \\
0 \\
0 \\
0 \\
0 \\
0\end{array}$ & $\begin{array}{r}98.1 \\
100.0 \\
100.0 \\
100.0 \\
100.0 \\
100.0 \\
98.4 \\
98.6 \\
100.0 \\
100.0 \\
97.8\end{array}$ & $\begin{array}{l}1.6 \\
0 \\
0 \\
0 \\
1.3 \\
0.9 \\
0 \\
1.4 \\
0.8 \\
0 \\
0\end{array}$ & $\begin{array}{l}88.7 \\
89.2 \\
87.3 \\
90.5 \\
85.2 \\
79.5 \\
83.7 \\
85.7 \\
86.7 \\
88.5 \\
85.7\end{array}$ & $\begin{array}{r}9.7 \\
10.8 \\
12.7 \\
9.5 \\
13.5 \\
19.6 \\
16.3 \\
12.9 \\
12.5 \\
11.5 \\
14.3\end{array}$ & $\begin{array}{r}40.0 \\
3.1 \\
9.5 \\
1.8 \\
8.3 \\
4.9 \\
6.5 \\
6.0 \\
2.9 \\
5.2 \\
6.5\end{array}$ & $\begin{array}{r}11.5 \\
47.2 \\
55.7 \\
38.4 \\
52.3 \\
70.9 \\
37.0 \\
34.2 \\
38.5 \\
58.9 \\
6.7\end{array}$ & $\begin{array}{l}48.5 \\
49.7 \\
34.8 \\
59.8 \\
39.5 \\
24.2 \\
56.5 \\
59.8 \\
58 .\end{array}$ & $\begin{array}{l}95.9 \\
72.7 \\
83.3 \\
62.5 \\
35.5 \\
87.5 \\
63.0 \\
91.7 \\
91.7 \\
52.4 \\
85.7\end{array}$ & $\begin{array}{r}2.4 \\
27.3 \\
16.7 \\
25.0 \\
61.3 \\
12.5 \\
25.9 \\
8.3 \\
8.3 \\
47.6 \\
14.3\end{array}$ & $\begin{array}{c}1.8 \\
0 \\
0 \\
12.5 \\
3.2 \\
0 \\
11.1 \\
0 \\
0 \\
0 \\
0\end{array}$ & $\begin{array}{l}50.5 \\
67.6 \\
47.0 \\
44.2 \\
52.0 \\
32.9 \\
64.1 \\
65.1\end{array}$ & $\begin{array}{l}47.6 \\
29.6 \\
48.5 \\
51.9 \\
43.2 \\
53.2 \\
32.5 \\
29.4 \\
24.4\end{array}$ & $\begin{array}{r}1.9 \\
2.8 \\
4.5 \\
3.8 \\
4.7 \\
13.9 \\
3.4 \\
5.5 \\
1.7 \\
3.4 \\
1.9\end{array}$ & $\begin{array}{l}73.5 \\
70.6 \\
52.1 \\
68.3 \\
68.4 \\
28.0 \\
66.0 \\
61.0 \\
65.4 \\
71.1 \\
55.2\end{array}$ & $\begin{array}{l}16.3 \\
25.3 \\
39.8 \\
26.9 \\
25.0 \\
57.8 \\
25.5 \\
35.3 \\
27.7 \\
25.1 \\
37.9\end{array}$ & $\begin{array}{r}10.2 \\
4.1 \\
8.1 \\
4.8 \\
6.6 \\
14.2 \\
8.5 \\
3.7 \\
6.9 \\
3.8 \\
6.9\end{array}$ \\
\hline $\begin{array}{l}126-793 \mathrm{~A}- \\
6 \mathrm{H}-2,79-82 \\
6 \mathrm{H}-3,44-48 \\
7 \mathrm{H}-1,94-98 \\
7 \mathrm{H}-2,37-40 \\
8 \mathrm{H}-1,32-36 \\
9 \mathrm{H}-2,111-114 \\
10 \mathrm{H}-2,65-68 \\
11 \mathrm{H}-4,44-47\end{array}$ & $\begin{array}{l}0.4 \\
1.5 \\
0.6 \\
2.3 \\
0 \\
0.2 \\
0.2 \\
1.2\end{array}$ & $\begin{array}{r}22.0 \\
8.8 \\
14.0 \\
19.0 \\
7.3 \\
9.2 \\
7.2 \\
9.7\end{array}$ & $\begin{array}{l}77.6 \\
89.7 \\
85.3 \\
78.6 \\
92.7 \\
90.6 \\
92.6 \\
89.1\end{array}$ & $\begin{array}{l}0 \\
2.4 \\
1.4 \\
7.2 \\
0 \\
0 \\
0 \\
9.6\end{array}$ & $\begin{array}{l}0 \\
0 \\
0 \\
0 \\
0 \\
0 \\
0 \\
0\end{array}$ & $\begin{array}{r}100.0 \\
97.6 \\
98.6 \\
92.8 \\
100.0 \\
100.0 \\
100.0 \\
90.4\end{array}$ & $\begin{array}{l}0 \\
0 \\
2.4 \\
1.5 \\
0 \\
0.8 \\
1.1 \\
0\end{array}$ & $\begin{array}{l}85.9 \\
81.6 \\
82.1 \\
75.4 \\
95.1 \\
92.4 \\
88.0 \\
81.8\end{array}$ & $\begin{array}{r}14.1 \\
18.4 \\
15.4 \\
23.1 \\
4.9 \\
6.8 \\
10.9 \\
18.2\end{array}$ & $\begin{array}{r}68.9 \\
23.0 \\
53.2 \\
27.1 \\
4.6 \\
5.2 \\
38.3 \\
8.5\end{array}$ & $\begin{array}{r}18.6 \\
6.3 \\
23.5 \\
33.5 \\
1.3 \\
9.3 \\
6.3 \\
6.0\end{array}$ & $\begin{array}{l}12.4 \\
70.7 \\
23.3 \\
39.4 \\
94.1 \\
85.4 \\
55.4 \\
8.1\end{array}$ & $\begin{array}{l}87.0 \\
98.8 \\
92.7\end{array}$ & $\begin{array}{r}6.1 \\
8.2 \\
13.5 \\
9.3 \\
9.5 \\
13.0 \\
0.6 \\
6.2\end{array}$ & $\begin{array}{l}3.3 \\
2.0 \\
2.8 \\
2.1 \\
0 \\
0 \\
0.6 \\
1.1\end{array}$ & $\begin{array}{l}74.4 \\
67.2 \\
62.9\end{array}$ & $\begin{array}{l}50.0 \\
21.5 \\
36.2 \\
33.3 \\
7.9 \\
23.7 \\
29.6 \\
28.6\end{array}$ & $\begin{array}{l}2.3 \\
4.0 \\
7.4 \\
6.4 \\
0.5 \\
1.9 \\
3.2 \\
8.6\end{array}$ & $\begin{array}{l}43.9 \\
59.3 \\
53.7 \\
49.2 \\
50.0 \\
56.1 \\
71.4 \\
84.6\end{array}$ & $\begin{array}{l}53.0 \\
25.9 \\
40.0 \\
35.0 \\
50.0 \\
41.5 \\
25.0 \\
11.5\end{array}$ & $\begin{array}{r}3.0 \\
14.8 \\
6.3 \\
15.8 \\
0 \\
2.4 \\
3.6 \\
3.8\end{array}$ \\
\hline
\end{tabular}

usually contain mixed glass colors. This heterogeneity may be a function of either eruptive mixing or mixing during transport.

These results suggest slight compositional differences between sands deposited in backarc and forearc intraoceanic settings. The volcaniclastic sands deposited in and adjacent to the Sumisu Rift contain higher percentages of felsic components (pumice and colorless glassy fragments, felsitic microcrystalline lithic fragments, and quartz) than do forearc sands. The localization of the pumiceous deposits is probably a function of wind and ocean currents that favor distribution to the west of the magmatic arc. Although the major source of volcaniclastic sand is the modern magmatic arc, intrarift mafic volcanic centers may also supply sediment to the Sumisu Rift basin. The unique tachylite-rich sand of Site 787 sand may be attributed to either winnowing by submarine-canyon currents or a volcanicisland source. More detailed volcanic provenance studies are required, such as the chemical fingerprinting of glass and crystal types (see articles by Fujioka and Rodolfo, this volume), in order to tie these grains precisely to their source volcanoes.

\section{ACKNOWLEDGMENTS}

This project was supported by a grant from the National Science Foundation and Joint Oceanographic Institutions administered by the Texas A\&M Research Foundation. The contents of this paper do not necessarily reflect the views of the National Science Foundation, the Joint Oceanographic Institutions, Inc., or the Texas A\&M University. I would like to thank Raymond V. Ingersoll, Greg Mack, and Michelle Seward for their thoughtful reviews of the text.

\section{REFERENCES}

Brown, G., and Taylor, B., 1988. Sea-floor mapping of the Sumisu Rift, Izu-Ogasawara (Bonin) Island Arc. Bull. Geol. Surv. Jpn., 39(1):23-38.

Cas, R. A., and Wright, J. V., 1987. Volcanic Successions: Modern and Ancient: A Geological Approach to Processes, Products and Successions: London (Allen and Unwin).

Dickinson, W. R., 1970. Interpreting detrital modes of graywacke and arkose. J. Sediment. Petrol., 40:695-707.

Dickinson, W. R., Beard, L. S., Brakenridge, G. R., Erjavec, J. L., Ferguson, R. C., Inman, K. F., Knepp, R. A., Lindberg, F. A., and Ryberg, P. T., 1983. Provenance of North American Phanerozoic sandstones in relation to tectonic setting. Geol. Soc. Am. Bull., 94:222-235.

Fisher, R. V., and Schmincke, H.-U., 1984. Pyroclastic Rocks: New York (Springer-Verlag).

Fryer, P., Langmuir, C., Taylor, B., Zhang, Y., and Hussong, D., 1985. Rifting of the Izu Arc. III. Relationship of chemistry to tectonics. Eos, 66:421.

Ingersoll, R. V., Bullard, T. F., Ford, R. L., Grimm, J. P., Pickle, J. D., and Sares, S. W., 1984. The effect of grain size on detrital modes: a test of the GazziDickinson point-counting method. J. Sediment. Petrol., 54:103-116.

Leg 126 Shipboard Scientific Party, 1989. Arc volcanism and rifting. Nature, 342:18-20.

Macdonald, G. A., 1972. Volcanoes: Englewood Cliffs, NJ (Prentice-Hall).

Marsaglia, K. M., 1989. Petrography, provenance, and diagenesis of arcrelated sands recovered by the Deep Sea Drilling Project on circumPacific, Mediterranean and Caribbean legs [Ph.D. dissert.]. Univ. California, Los Angeles.

, 1991. Provenance of sands and sandstones from a rifted continental arc, Gulf of California, Mexico. In Fisher, R. V., and Smith, G. A. (Eds.), Sedimentation in Volcanic Settings. Spec. Publ., Soc. Econ. Paleontol. Mineral., 45:237-248. 


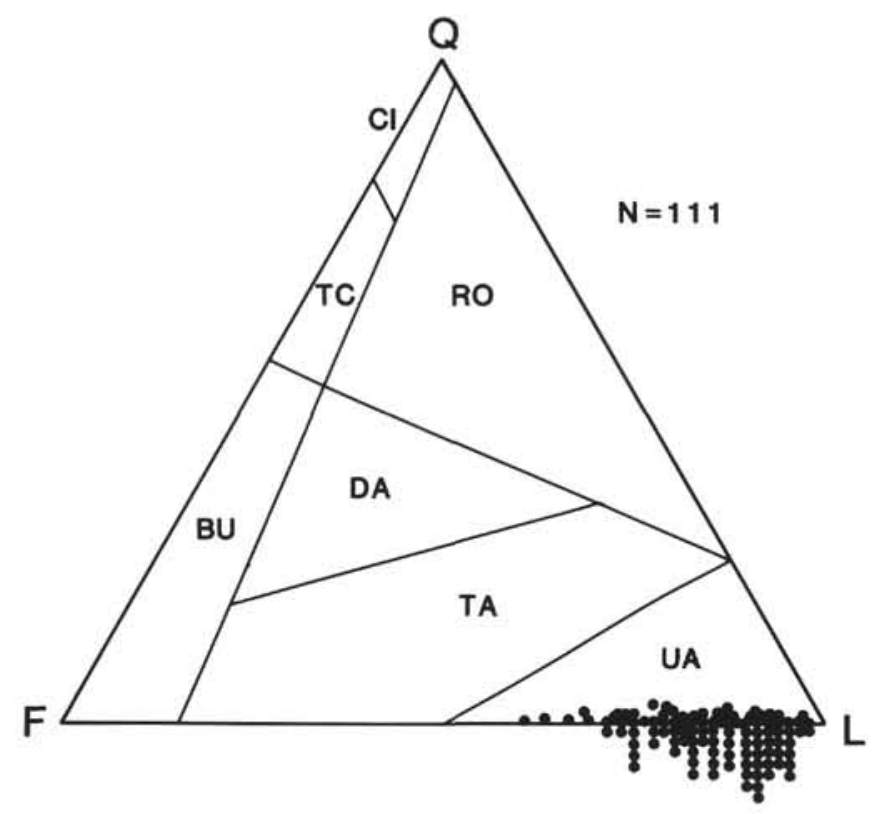

Figure 4. QFL plot of all Leg 126 samples. Overlapping data at base of diagram plotted in histogram fashion. Provenance fields are from Dickinson et al. (1983): $\mathrm{CI}=$ craton interior, $\mathrm{TC}=$ transitional continental, $\mathrm{BU}=$ basement uplift, $\mathrm{RO}=$ recycled orogen, $\mathrm{DA}=$ dissected $\operatorname{arc}, \mathrm{TA}=$ transitional arc, and $\mathrm{UA}=$ undissected arc.

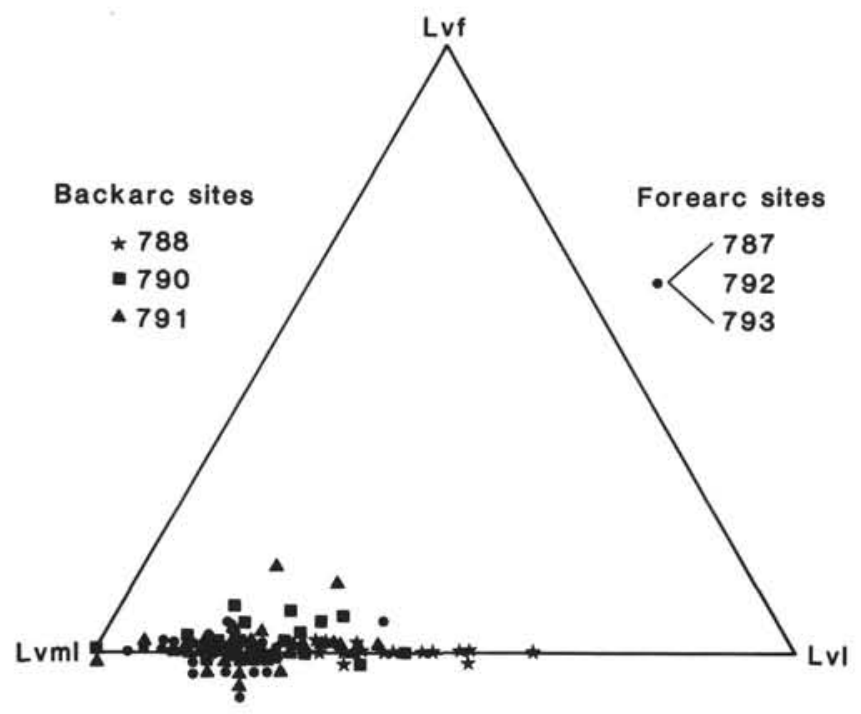

Figure 5. LvflvmlLvl ternary plot of backarc and forearc sites. Data for backarc sites are shown separately. Overlapping data along the base of the diagram have been plotted in histogram fashion. Lvf $=$ volcanic lithic with felsitic texture, $\mathrm{Lvml}=$ volcanic lithic with microlitic texture, and Lvl = volcanic lithic with lathwork texture. See text for discussion.

Natland, J. H., and Tarney, J., 1981. Petrologic evolution of the Mariana arc and back-arc basin system-a synthesis of drilling results in the South Philippine Sea. In Hussong, D. M., Uyeda, S., et al., Init. Repts. DSDP, 60: Washington (U.S. Govt. Printing Office), 877-908.

Nishimura, A., and Murakami, F., 1988. Sedimentation of the Sumisu Rift, Izu-Ogasawara Arc. Bull. Geol. Surv. Jpn., 39(1):39-61.

Nishimura, A., Marsaglia, K. M., Rodolfo, K. S., Colella, A., Hiscott, R. N., Tazaki, K., Gill, J. B., Janecek, T., Firth, J., Isiminger-Kelso, M.,

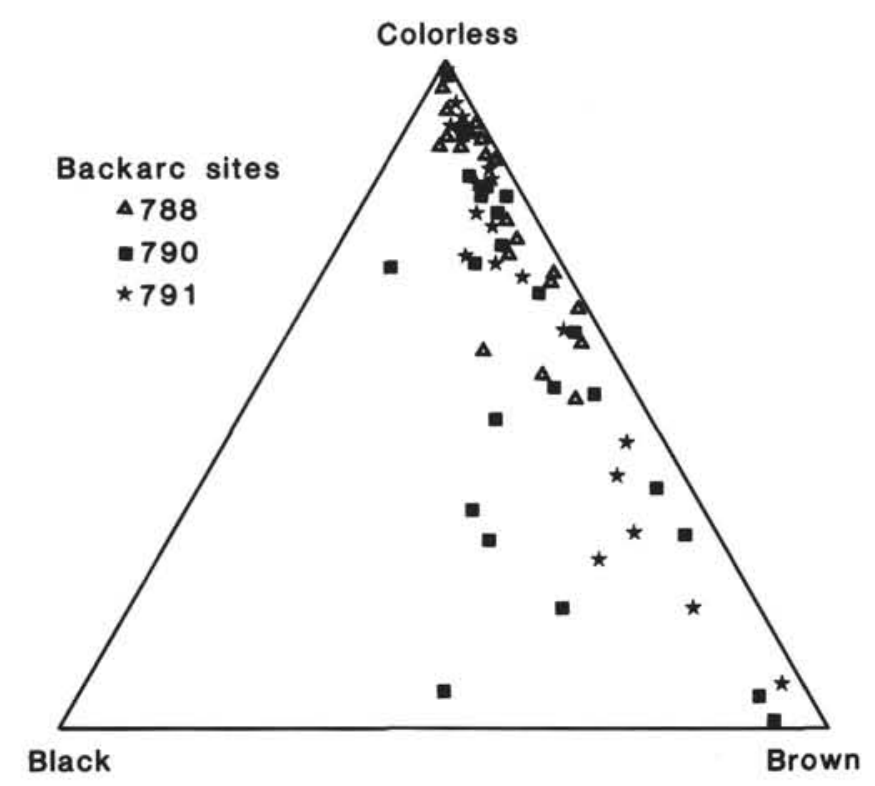

Figure 6. Triangular plot of glass proportions (colorless vs. black vs. brown) for backarc sites.

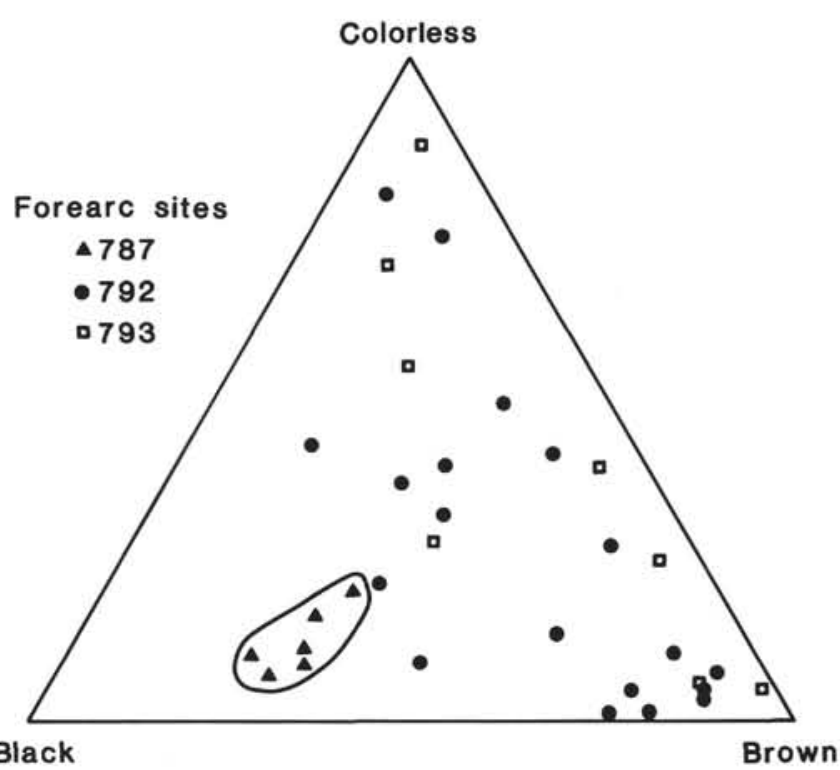

Figure 7. Triangular plot of glass proportions (colorless vs. black vs. brown) for forearc sites.

Herman, Y., Taylor, R. N., Taylor, B., Fujioka, K., and Leg 126 Scientific Party, 1991. Pliocene-Quaternary submarine pumice deposits in the Sumisu Rift area, Izu-Bonin Arc. In Fisher, R. V., and Smith, G. A. (Eds.), Sedimentation in Volcanic Settings. Spec. Publ., Soc. Econ. Paleontol. Mineral., 45:201-208.

Packer, B. M., and Ingersoll, R. V., 1986. Provenance and petrology of Deep Sea Drilling project sands and sandstones from the Japan and Mariana forearc and backarc regions. Sediment. Geol., 51:5-28.

Schmincke, H.-U., 1981. Ash from vitric muds in deep sea cores from the Mariana Trough and fore-arc region (South Philippine Sea) (Sites 453, 454, $455,458,459$, and SP), Deep Sea Drilling Project Leg 60. In Hussong, D. M., Uyeda, S., et al., Init. Repts. DSDP, 60: Washington (U.S. Govt. Printing Office), 473-481.

Shipboard Scientific Party, 1981a. Site 453: west side of the Mariana Trough. 


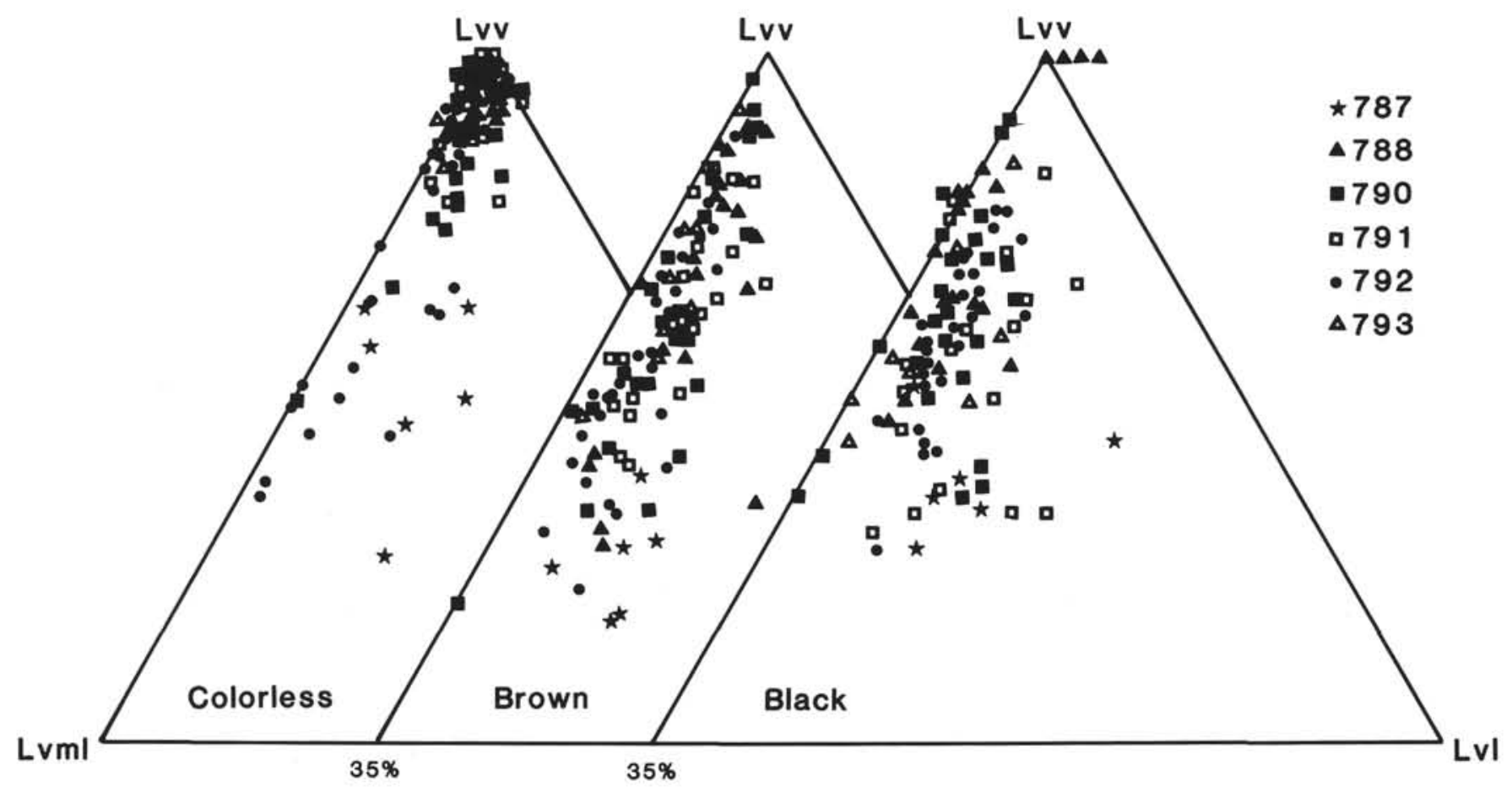

Figure 8. LvvLvmlLvl plots for black, brown, and colorless glassy volcanic lithic grains. These plots give some indication of the degree of crystallinity of the various glass types.

Table 4. Mean recalculated parameters.

\begin{tabular}{|c|c|c|c|c|c|c|c|c|c|c|c|c|c|c|c|c|c|c|c|c|c|c|c|}
\hline \multirow[b]{2}{*}{ Holes } & \multirow[b]{2}{*}{ Age } & \multirow[b]{2}{*}{$N$} & \multicolumn{3}{|c|}{ QFL\% } & \multicolumn{3}{|c|}{ QmKP\% } & \multicolumn{3}{|c|}{ LvfLvmILvi\% } & \multicolumn{3}{|c|}{ VolcTor\% } & \multicolumn{3}{|c|}{$\begin{array}{c}\text { Clear } \\
\text { LvvLvmlLvvl\% }\end{array}$} & \multicolumn{3}{|c|}{$\begin{array}{c}\text { Brown } \\
\text { LwvLvmlLvvi\% }\end{array}$} & \multicolumn{3}{|c|}{$\begin{array}{c}\text { Black } \\
\text { LvvLvm|Lvvi\% }\end{array}$} \\
\hline & & & 0 & $\mathbf{F}$ & $\mathrm{L}$ & $\mathrm{Om}$ & $\mathrm{K}$ & $\mathrm{P}$ & Lvf & Lvml & ivl & clgl & bigl & brgl & Lw & Lvml & Lvl & Lvv & Lvml & Lvi & Lw & Lvml & LvI \\
\hline 787 A and 787B & Quaternary & ${ }^{6 \text { Avg }}$ & $\begin{array}{l}0.6 \\
0.4\end{array}$ & $\begin{array}{r}24.7 \\
4.4\end{array}$ & $\begin{array}{r}74.8 \\
4.3\end{array}$ & $\begin{array}{l}0.6 \\
0.6\end{array}$ & $\begin{array}{l}0 \\
0\end{array}$ & $\begin{array}{l}99.5 \\
0.6\end{array}$ & $\begin{array}{l}1.8 \\
1.5\end{array}$ & $\begin{array}{c}70.1 \\
8.1\end{array}$ & $\begin{array}{r}28.1 \\
6.8\end{array}$ & $\begin{array}{r}12.1 \\
4.4\end{array}$ & $\begin{array}{r}58.6 \\
6.3\end{array}$ & $\begin{array}{r}29.4 \\
2.8\end{array}$ & $\begin{array}{l}51.2 \\
12.4\end{array}$ & $\begin{array}{l}34.7 \\
8.9\end{array}$ & $\begin{array}{r}14.1 \\
7.4 \\
\end{array}$ & $\begin{array}{r}26.2 \\
7.0\end{array}$ & $\begin{array}{r}56.6 \\
6.4\end{array}$ & $\begin{array}{r}17.2 \\
4.4\end{array}$ & $\begin{array}{l}34.9 \\
10.1\end{array}$ & $\begin{array}{r}44.3 \\
4.0\end{array}$ & $\begin{array}{r}20.8 \\
8.9\end{array}$ \\
\hline $788 \mathrm{~A}$ and $788 \mathrm{C}$ & Quaternary & ${ }^{3 \text { Avgs }}$ & $\begin{array}{l}0.4 \\
0.6\end{array}$ & $\begin{array}{r}23.5 \\
2.4\end{array}$ & $\begin{array}{r}76.1 \\
2.9\end{array}$ & $\begin{array}{l}1.1 \\
1.6\end{array}$ & $\begin{array}{l}0 \\
0\end{array}$ & $\begin{array}{r}98.9 \\
1.6\end{array}$ & $\begin{array}{l}0.6 \\
0.8\end{array}$ & $\begin{array}{r}66.4 \\
1.6\end{array}$ & $\begin{array}{r}33.1 \\
1.9\end{array}$ & $\begin{array}{l}71.0 \\
12.8\end{array}$ & $\begin{array}{l}2.2 \\
0.9\end{array}$ & $\begin{array}{l}26.7 \\
12.1\end{array}$ & $\begin{array}{r}92.9 \\
2.2\end{array}$ & $\begin{array}{l}3.5 \\
1.1\end{array}$ & $\begin{array}{l}3.6 \\
2.2\end{array}$ & $\begin{array}{r}71.0 \\
4.7\end{array}$ & $\begin{array}{r}20.1 \\
3.8\end{array}$ & $\begin{array}{l}8.9 \\
4.0\end{array}$ & $\begin{array}{l}78.2 \\
18.6\end{array}$ & $\begin{array}{l}18.8 \\
14.9\end{array}$ & $\begin{array}{l}3.0 \\
4.3\end{array}$ \\
\hline $788 \mathrm{C}$ & Pliocene & 18 Avg & $\begin{array}{l}0.1 \\
0.1\end{array}$ & $\begin{array}{l}16.7 \\
10.9\end{array}$ & $\begin{array}{l}88.2 \\
10.9\end{array}$ & $\begin{array}{l}0.4 \\
0.6\end{array}$ & $\begin{array}{l}0 \\
0\end{array}$ & $\begin{array}{r}99.6 \\
0.6\end{array}$ & $\begin{array}{l}0.4 \\
0.9\end{array}$ & $\begin{array}{l}62.6 \\
13.3\end{array}$ & $\begin{array}{r}37.0 \\
13.7\end{array}$ & $\begin{array}{l}77.7 \\
14.5\end{array}$ & $\begin{array}{l}4.6 \\
4.1\end{array}$ & $\begin{array}{l}17.7 \\
12.4\end{array}$ & $\begin{array}{r}94.6 \\
2.4\end{array}$ & $\begin{array}{l}2.3 \\
1.4\end{array}$ & $\begin{array}{l}3.1 \\
1.7\end{array}$ & $\frac{64.8}{20.8}$ & $\begin{array}{l}27.7 \\
16.9\end{array}$ & 7.5 & $\begin{array}{l}72.2 \\
16.1\end{array}$ & $\begin{array}{l}23.8 \\
13.3\end{array}$ & $\begin{array}{l}4.0 \\
4.9\end{array}$ \\
\hline $790 \mathrm{~A}, 790 \mathrm{~B}$ and $790 \mathrm{C}$ & Quaternary & 25 Avg & $\begin{array}{l}0.9 \\
0.7\end{array}$ & $\begin{array}{r}14.6 \\
6.9\end{array}$ & $\begin{array}{r}84.5 \\
7.1\end{array}$ & $\begin{array}{l}2.5 \\
2.9\end{array}$ & $\begin{array}{l}0 \\
0.2\end{array}$ & $\begin{array}{r}97.5 \\
3.0\end{array}$ & $\begin{array}{l}2.0 \\
2.3\end{array}$ & $\begin{array}{r}75.8 \\
9.8\end{array}$ & $\frac{2.2}{9.4}$ & $\begin{array}{l}55.5 \\
28.5\end{array}$ & $\begin{array}{l}11.0 \\
11.4\end{array}$ & $\begin{array}{l}33.5 \\
24.1\end{array}$ & $\begin{array}{l}86.8 \\
11.1\end{array}$ & $\begin{array}{r}9.8 \\
10.5\end{array}$ & $\begin{array}{l}3.4 \\
2.6\end{array}$ & $\begin{array}{l}58.5 \\
17.8\end{array}$ & $\begin{array}{l}34.9 \\
16.8\end{array}$ & $\begin{array}{l}6.5 \\
4.8\end{array}$ & $\begin{array}{l}61.7 \\
15.2\end{array}$ & $\begin{array}{l}31.11 \\
12.4\end{array}$ & 7.2 \\
\hline $791 \mathrm{~A}$ and $791 \mathrm{~B}$ & Quaternary & $\begin{array}{l}22 \text { Avg } \\
\text { Std }\end{array}$ & $\begin{array}{l}0.5 \\
0.4\end{array}$ & $\begin{array}{c}11.2 \\
7.1\end{array}$ & $\begin{array}{r}88.2 \\
7.3\end{array}$ & $\begin{array}{l}2.3 \\
3.0\end{array}$ & $\begin{array}{l}0.3 \\
0.8\end{array}$ & $\begin{array}{r}97.5 \\
3.3\end{array}$ & $\begin{array}{l}2.1 \\
3.6\end{array}$ & $\begin{array}{r}75.4 \\
9.9\end{array}$ & $\begin{array}{l}22.6 \\
9.1\end{array}$ & $\begin{array}{l}66.4 \\
26.6\end{array}$ & $\begin{array}{l}5.8 \\
3.9\end{array}$ & $\begin{array}{l}27.8 \\
24.7\end{array}$ & $\begin{array}{r}92.2 \\
5.9\end{array}$ & $\begin{array}{l}5.4 \\
4.0\end{array}$ & $\begin{array}{l}2.5 \\
2.8\end{array}$ & $\begin{array}{l}64.6 \\
15.1\end{array}$ & $\begin{array}{l}28.7 \\
13.1\end{array}$ & $\begin{array}{l}6.7 \\
4.4\end{array}$ & $\begin{array}{l}54.5 \\
15.1\end{array}$ & $\begin{array}{l}31.9 \\
13.9\end{array}$ & $\begin{array}{l}13.6 \\
11.5\end{array}$ \\
\hline $792 A$ and $792 B$ & Quaternary & 17 Avg & $\begin{array}{l}0.5 \\
0.4\end{array}$ & $\begin{array}{r}13.0 \\
6.8\end{array}$ & $\begin{array}{r}86.4 \\
6.9\end{array}$ & $\begin{array}{l}2.0 \\
1.8\end{array}$ & $\begin{array}{l}0.4 \\
1.1\end{array}$ & $\begin{array}{r}97.5 \\
2.3\end{array}$ & 1.0 & $\begin{array}{l}82.0 \\
4.0\end{array}$ & $\begin{array}{r}16.9 \\
4.3\end{array}$ & $\begin{array}{l}25.8 \\
23.6\end{array}$ & $\frac{2.7}{12.1}$ & $\begin{array}{l}51.5 \\
25.6\end{array}$ & $\begin{array}{l}74.3 \\
18.6\end{array}$ & $\begin{array}{l}21.9 \\
16.7\end{array}$ & $\begin{array}{l}3.8 \\
4.1\end{array}$ & $\begin{array}{l}52.9 \\
15.7\end{array}$ & $\begin{array}{l}40.3 \\
14.3\end{array}$ & $\begin{array}{l}6.8 \\
2.8\end{array}$ & $\begin{array}{l}59.7 \\
11.3\end{array}$ & $\begin{array}{l}32.1 \\
9.9\end{array}$ & $\begin{array}{l}8.2 \\
3.5\end{array}$ \\
\hline $792 \mathrm{~B}$ and $792 \mathrm{E}$ & Pliocene & ${ }^{3 \text { Avg }}$ & $\begin{array}{l}0.6 \\
0.4\end{array}$ & $\begin{array}{r}18.3 \\
5.8\end{array}$ & $\begin{array}{r}81.1 \\
5.7\end{array}$ & $\begin{array}{l}1.0 \\
0.8\end{array}$ & $\begin{array}{l}0 \\
0\end{array}$ & $\begin{array}{r}99.0 \\
0.8\end{array}$ & $\begin{array}{l}1.0 \\
0.7\end{array}$ & $\begin{array}{l}85.9 \\
4.4\end{array}$ & $\begin{array}{r}13.1 \\
4.1\end{array}$ & $\begin{array}{l}23.8 \\
15.4\end{array}$ & $\begin{array}{l}38.8 \\
19.8\end{array}$ & $\begin{array}{l}37.4 \\
16.6\end{array}$ & $\begin{array}{l}68.8 \\
23.9\end{array}$ & $\begin{array}{l}29.6 \\
23.2\end{array}$ & $\begin{array}{l}1.6 \\
1.3\end{array}$ & $\begin{array}{l}52.6 \\
11.5\end{array}$ & $\begin{array}{l}40.3 \\
7.7\end{array}$ & $\begin{array}{l}7.1 \\
6.8\end{array}$ & $\begin{array}{l}62.3 \\
13.9\end{array}$ & $\begin{array}{l}28.3 \\
11.2\end{array}$ & $\begin{array}{l}9.5 \\
4.1\end{array}$ \\
\hline $792 \mathrm{E}$ & Miocene & ${ }^{9} \mathrm{Avg}$ & 0.2 & $\begin{array}{r}13.9 \\
4.2\end{array}$ & $\begin{array}{r}85.9 \\
4.0\end{array}$ & $\begin{array}{l}0.6 \\
0.8\end{array}$ & $\begin{array}{l}0 \\
0\end{array}$ & $\begin{array}{r}99.4 \\
0.8\end{array}$ & $\begin{array}{l}0.5 \\
0.6\end{array}$ & $\begin{array}{r}85.9 \\
2.9\end{array}$ & $\begin{array}{r}13.6 \\
2.7\end{array}$ & $\begin{array}{l}5.7 \\
2.3\end{array}$ & $\begin{array}{l}43.6 \\
17.5\end{array}$ & $\begin{array}{l}50.7 \\
17.8\end{array}$ & $\begin{array}{l}72.6 \\
18.9\end{array}$ & $\begin{array}{l}24.4 \\
17.4\end{array}$ & $\begin{array}{l}3.0 \\
4.8\end{array}$ & $\begin{array}{l}59.8 \\
16.2\end{array}$ & $\begin{array}{l}35.5 \\
13.8\end{array}$ & $\begin{array}{l}4.8 \\
3.4\end{array}$ & $\begin{array}{l}59.5 \\
12.6\end{array}$ & $\begin{array}{l}33.4 \\
10.2\end{array}$ & $\begin{array}{l}7.1 \\
3.0\end{array}$ \\
\hline $793 \mathrm{~A}$ & Quaternary & 8 Avg & $\begin{array}{l}0.8 \\
0.7\end{array}$ & $\frac{12.2}{5.3}$ & $\begin{array}{r}87.0 \\
5.6\end{array}$ & $\begin{array}{l}2.6 \\
3.5\end{array}$ & $\begin{array}{l}0 \\
0\end{array}$ & $\begin{array}{r}97.4 \\
3.5\end{array}$ & $\begin{array}{l}0.7 \\
0.8\end{array}$ & $\begin{array}{r}85.3 \\
6.0\end{array}$ & $\begin{array}{r}14.0 \\
5.8\end{array}$ & $\begin{array}{l}38.3 \\
27.4\end{array}$ & $\begin{array}{l}13.1 \\
10.3\end{array}$ & $\begin{array}{l}48.6 \\
30.9\end{array}$ & $\frac{90.2}{4.1}$ & $\begin{array}{l}8.3 \\
3.9\end{array}$ & $\begin{array}{l}1.5 \\
1.2\end{array}$ & $\begin{array}{l}66.9 \\
12.6\end{array}$ & $\begin{array}{l}28.9 \\
11.4\end{array}$ & $\begin{array}{l}4.3 \\
2.7\end{array}$ & $\begin{array}{l}58.5 \\
12.5\end{array}$ & $\begin{array}{l}35.2 \\
13.0\end{array}$ & $\begin{array}{l}6.2 \\
5.5\end{array}$ \\
\hline
\end{tabular}

In Hussong, D. M., Uyeda, S., et al., Init. Repts. DSDP, 60: Washington (U.S. Govt. Printing Office), 101-167.

1981b. Site 457: Mariana Island Arc, In Hussong, D. M., Uyeda, S., et al., Init. Repts. DSDP, 60: Washington (U.S. Govt. Printing Office), 255-261.

Taylor, B., Fujioka, K., et al., 1990. Proc. ODP, Init. Repts., 126: College Station, TX (Ocean Drilling Program).
Van der Plas, L., and Tobi, A. C., 1965. A chart for judging the reliability of point counting results. Am. J. Sci., 263:87-90.

Date of initial receipt: 2 January 1991

Date of acceptance: 28 May 1991

Ms 126B-124 


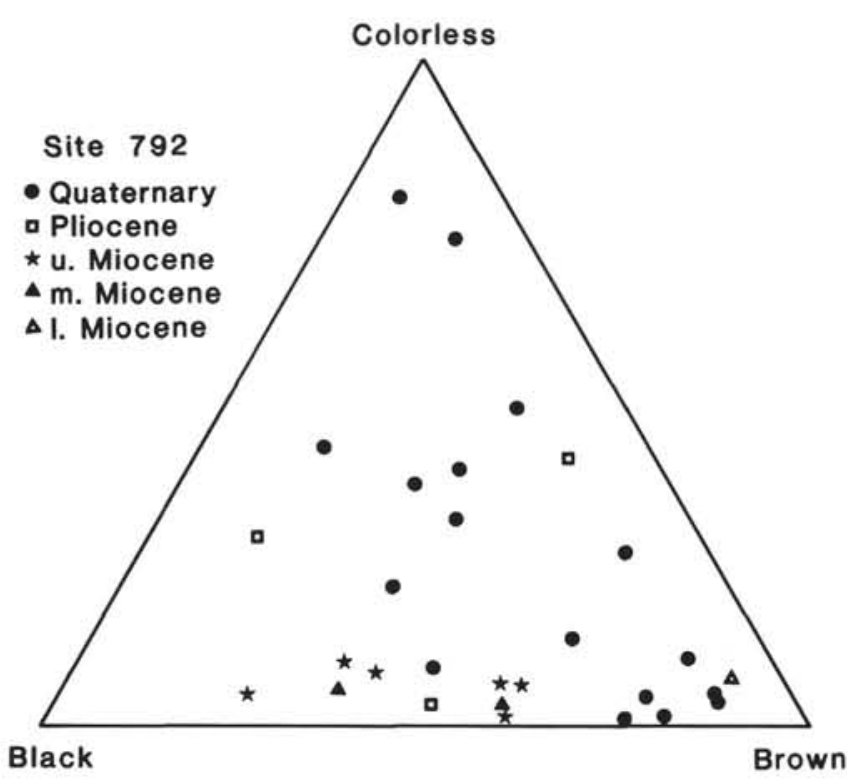

Figure 9. Ternary plot of glass proportions for Site 792 samples by age.

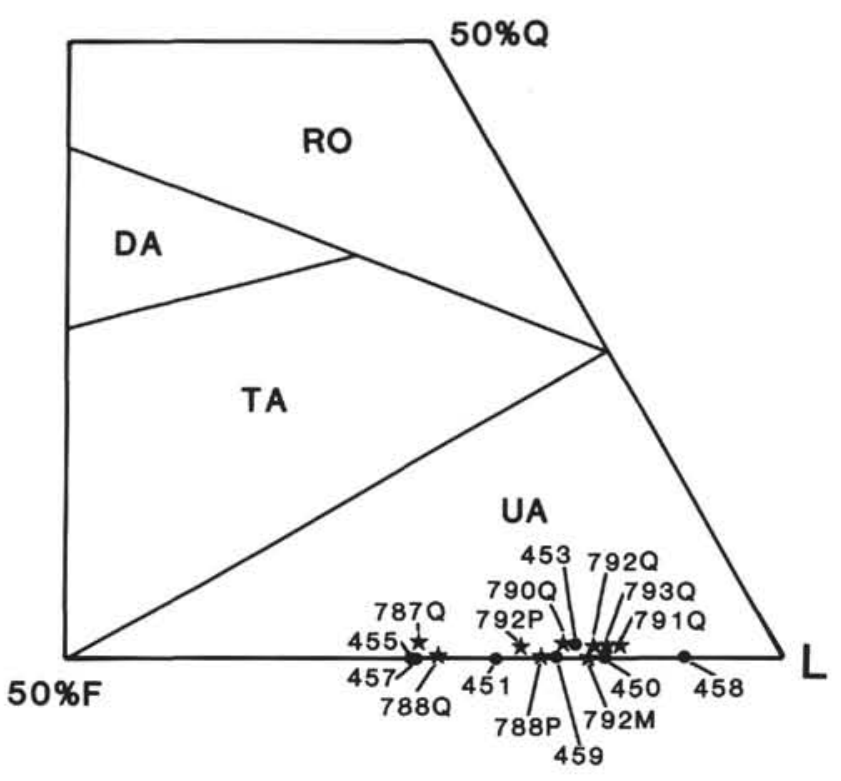

Figure 10. Enlargement of lithic corner of QFL diagram, where Mariana transect site means from Packer and Ingersoll (1986) and Leg 126 site means (Table 4) are plotted. Leg 126 means have been subdivided by age (i.e., 792Q $=$ Quaternary, $792 \mathrm{P}=$ Pliocene, and $792 \mathrm{M}=$ Miocene). Provenance fields are from Dickinson et al. (1983): $\mathrm{RO}=$ recycled orogen, $\mathrm{DA}=$ dissected arc, TA $=$ transitional arc, and $\mathrm{UA}=$ undissected arc. 


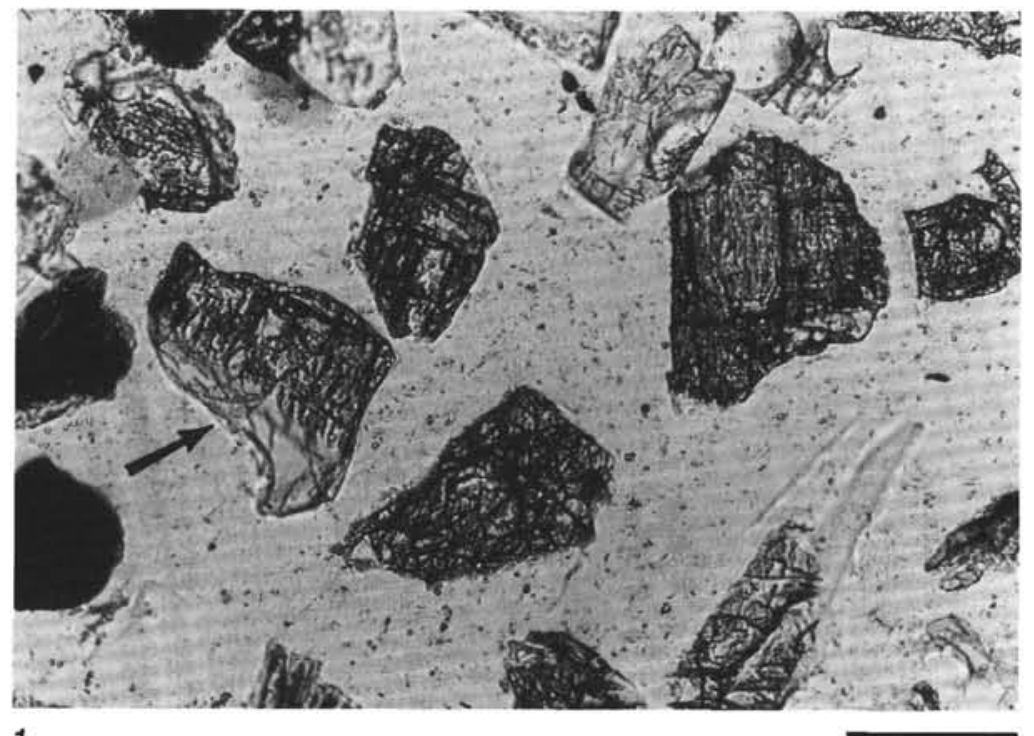

1

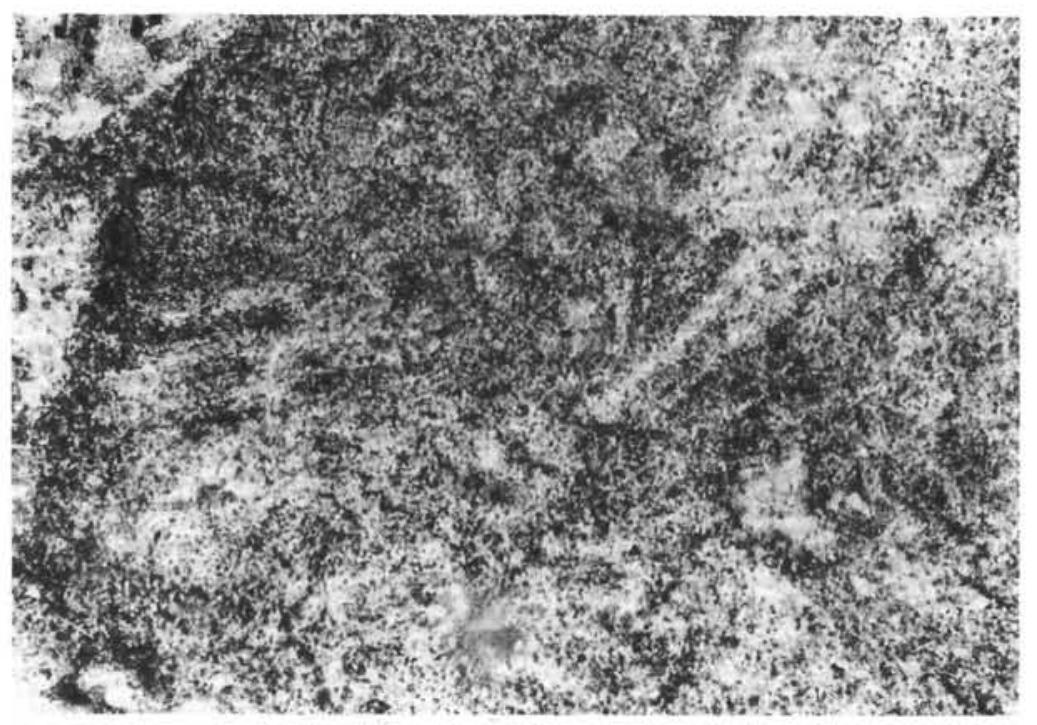

3
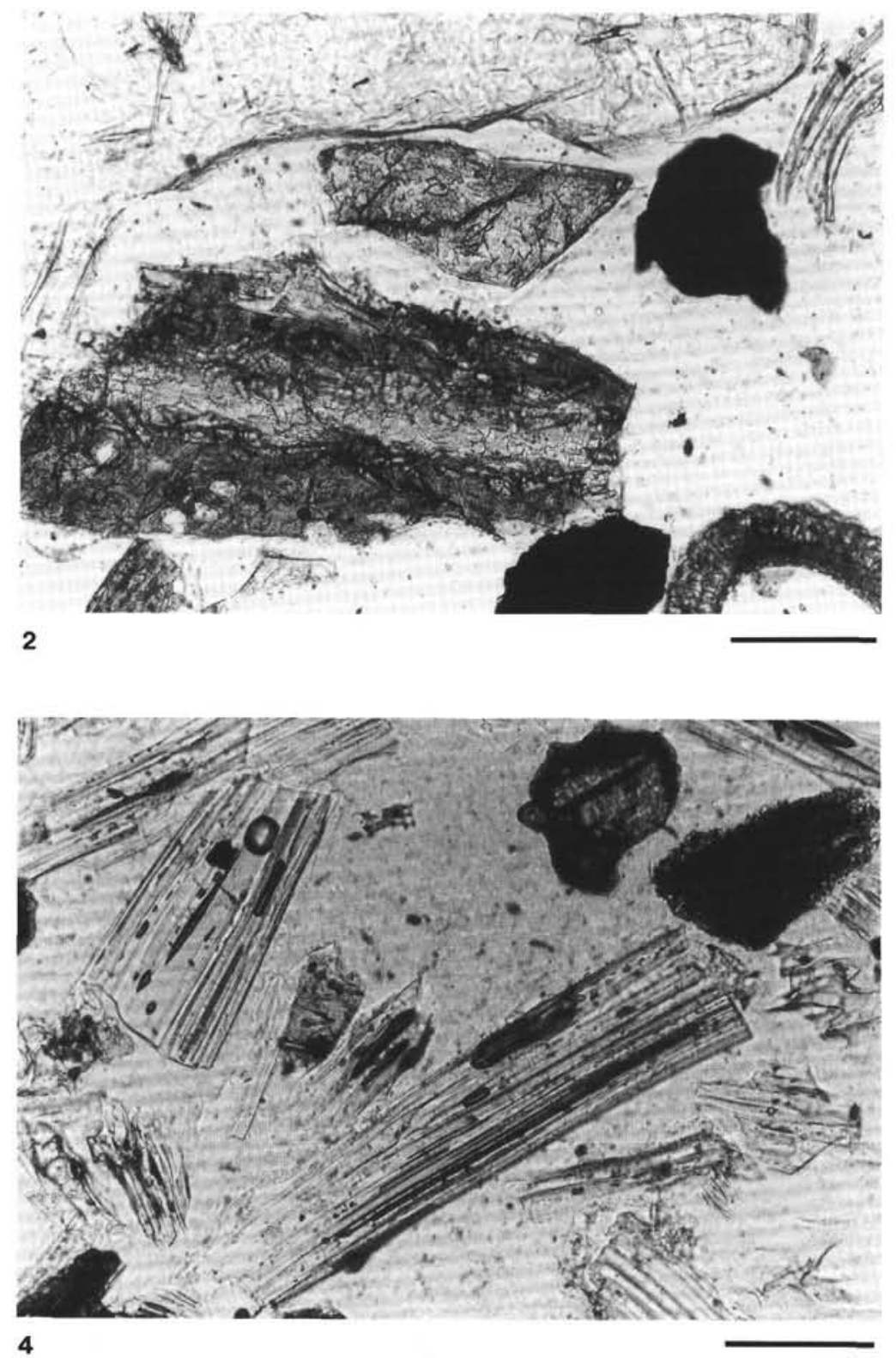

Plate 1. Photomicrographs, all plane-polarized light. Scale bars $=0.1 \mathrm{~mm}$. 1. Stained/etched plagioclase fragments, one in a colorless lathwork grain (arrow). Sample $126-788 \mathrm{C}-13 \mathrm{H}-5,120-124 \mathrm{~cm}$. 2. Various stained/etched grains, including a large brown, plagioclase-bearing, lathwork grain (lower center); a large colorless, nonvesicular glass fragment (top); and smaller grains of opaque dense minerals and plagioclase. Sample 126-790B-5H-1,113-116 cm. 3. Photomicrograph of stained/etched felsitic grain; grain fills entire field of view. Sample 126-791A-28X-CC, 8-11 cm. 4. Stretched or "woody" colorless pumice fragments in unstained part of slide. Minor black glass; one fragment is pyroxene-bearing. Sample 126-791 A-18H-2, 54-57 cm. 

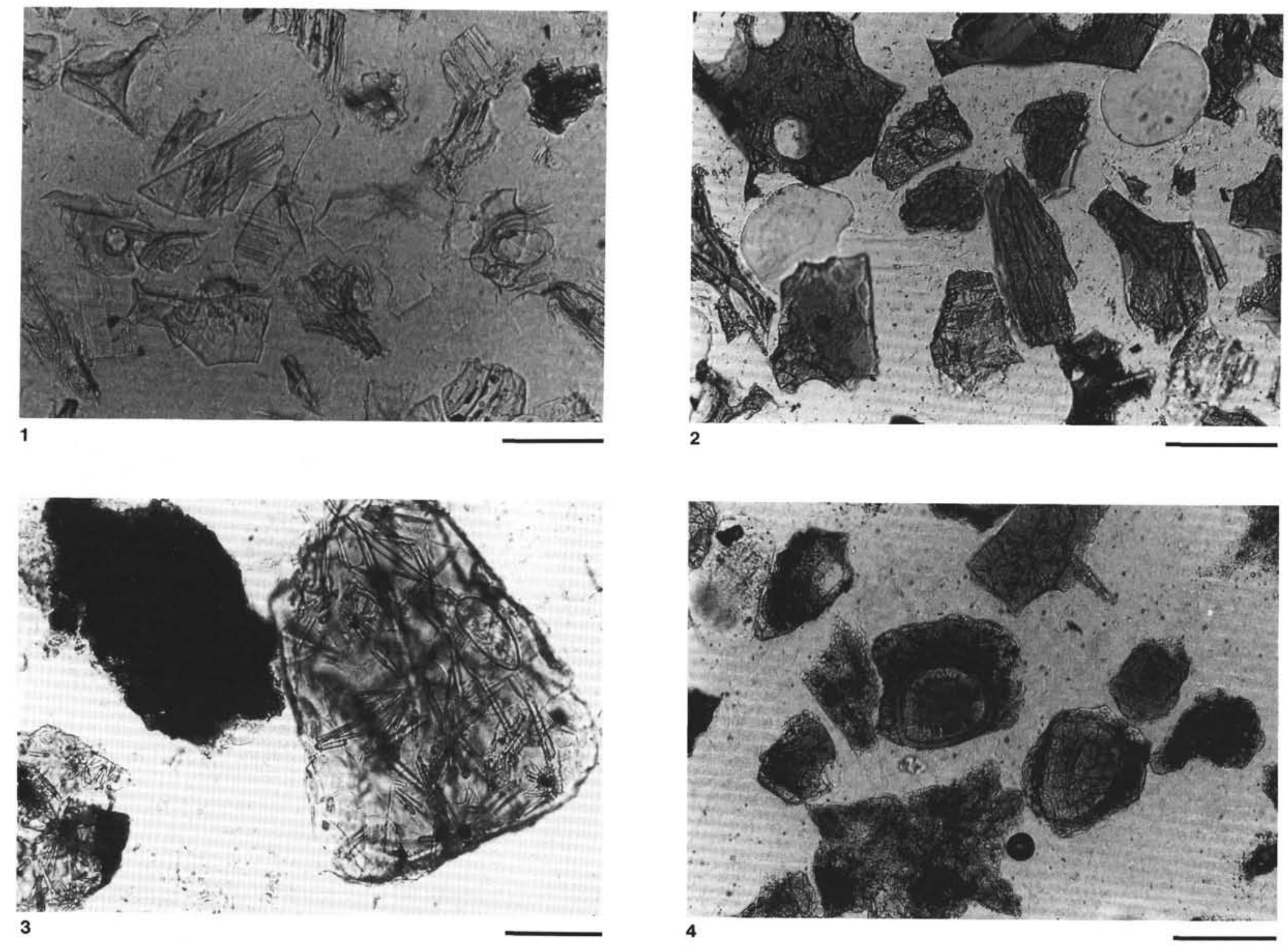

Plate 2. Photomicrographs, all plane-polarized light. Scale bars $=0.1 \mathrm{~mm}$, unless otherwise indicated. 1. Colorless glass shards (including bubble-wall and pumice fragments) in etched/stained part of slide. Sample 126-790B-15X-1, 52-55 cm. 2. Blocky to slightly vesicular brown glass fragments in etched/stained part of slide. A few fragments contain plagioclase crystals (light gray). Sample 126-793A-9H-2, $111-114 \mathrm{~cm}$. 3. Brown microlitic fragments and black vitric (tachylitic) grain. Note the irregular grain boundaries and slightly transparent edges of tachylite grain (these are characteristic of tachylite grains in Le 126 samples). Brown glass contains pyroxene microlites. Sample 126-790B-15X-1, 52-55 cm. 4. Glass fragments altered to clay minerals in etched/stained part of slide. Circular structures are vesicles filled with zoned clay minerals. Sample 126-791B-75R-1, 75-78 cm. 

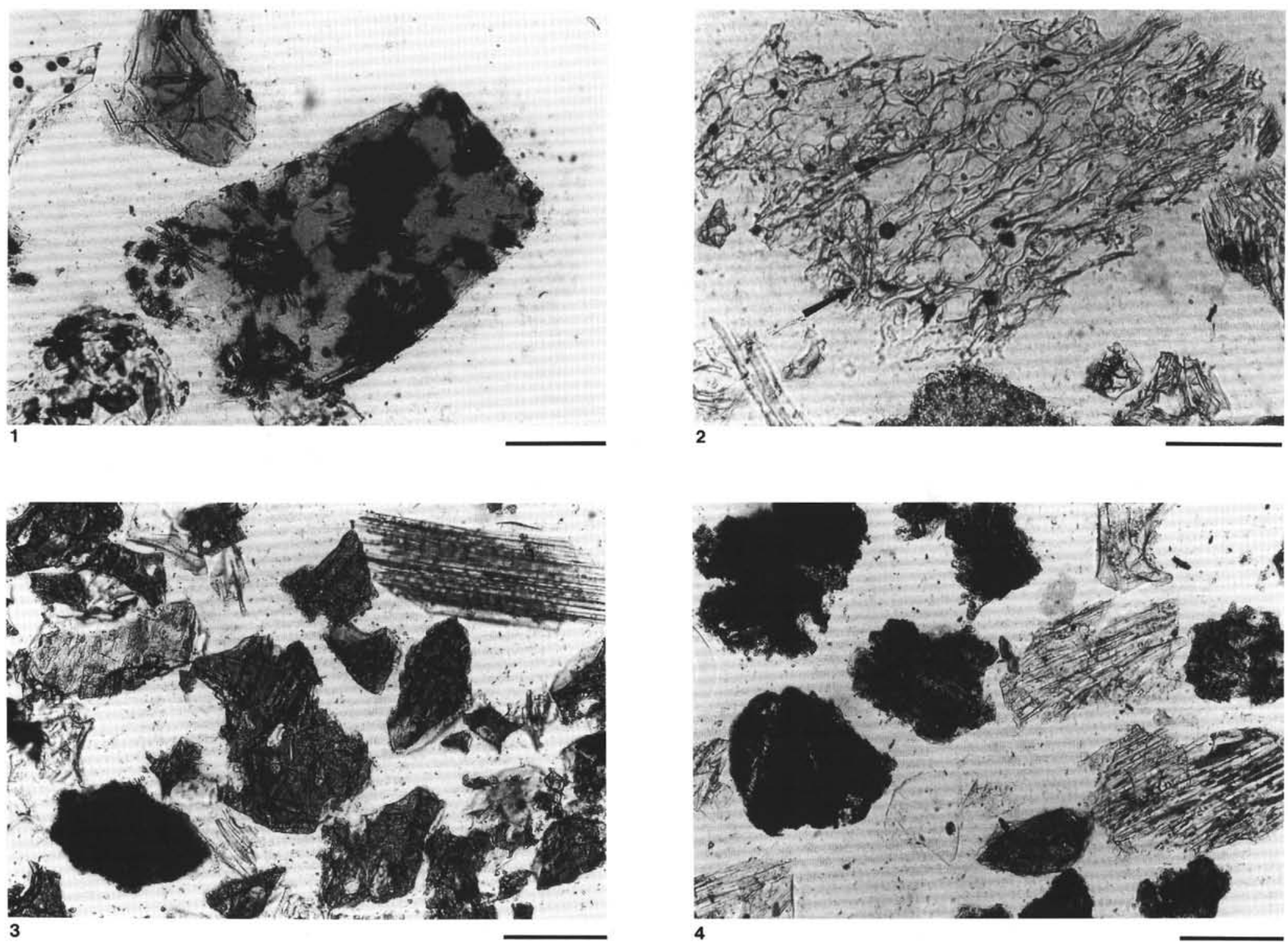

Plate 3. Photomicrographs, all plane-polarized light. Scale bars $=0.1 \mathrm{~mm}$, unless otherwise indicated. 1. Mixed brown/black (partially tachylitized) microlitic grain and brown/tan microlitic grains in unstained part of slide. Sample 126-788C-8H-2, 107-110 cm. Scale bar $=0.05 \mathrm{~mm}$. 2. Colorless microlitic pumice fragment in etched/stained part of slide. Microlites are best defined under crossed nicols, but some are distinguishable in plane light (arrow). Sample 126-791A-22H-5, 128-131 cm. 3. Heterogeneous volcaniclastic sand in etched/stained part of slide. Fragments of dark-brown glass dominate, with minor "woody" pumice and black vitric grains. Sample 126-793A-10H-2, 65-68 cm. 4. Heterogeneous volcaniclastic sand with black (tachylitic) vitric and microlitic grains, colorless glass bubble-wall and pumice fragments, and minor dark-brown glass fragments. Etched/stained part of slide. Sample 126-790C-12X-1, 81-84 cm. 

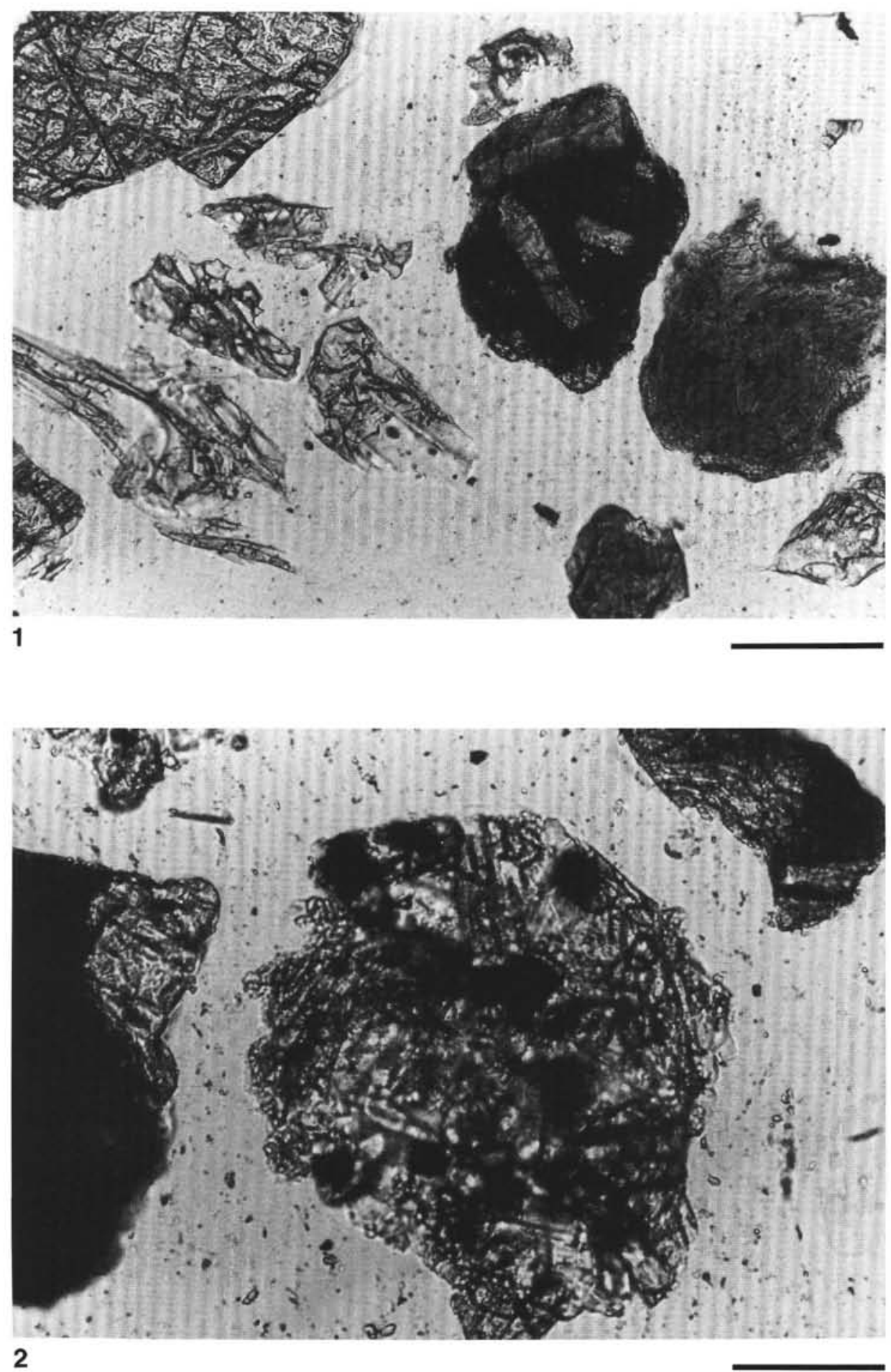

Plate 4. Photomicrographs, all plane-polarized light. 1. Black lathwork, brown vitric, clear vitric (irregular grain, left center), and plagioclase grain (upper left corner) in etched/stained part of slide. Sample 126-790B-10H-6, 119-120 cm. Scale bar $=0.1 \mathrm{~mm}$. 2. "Other volcanic lithic" grain composed of plagioclase, pyroxene, and opaque microlites (holocrystalline) in etched/stained part of slide. Black microlitic grains are located along edge of field of view. Sample $126-787 \mathrm{~A}-1 \mathrm{~W}-3,15-21 \mathrm{~cm}$. Scale bar $=0.05 \mathrm{~mm}$. 